\title{
Synthesis, Characterization and Biological Application of Pyrazolo[1,5-a]pyrimidine Based Organometallic $\operatorname{Re}(\mathrm{I})$ Complexes
}

\author{
Reena R. Varma, ${ }^{1}$ Juhee G. Pandya, ${ }^{2}$ Foram U. Vaidya, ${ }^{3}$ Chandramani Pathak, ${ }^{3}$ \\ Bhupesh S. Bhatt ${ }^{1}$ and Mohan N. Patel ${ }^{1, *}$ \\ ${ }^{1}$ Department of Chemistry, Sardar Patel University, Vallabh Vidyanagar-388 120, Gujarat (INDIA) \\ ${ }^{2}$ Department of Biosciences, Sardar Patel University, Vallabh Vidyanagar, Gujarat, (INDIA) \\ ${ }^{3}$ Department of Cell Biology, School of Biological Sciences and Biotechnology, Indian Institute of Advanced Research, Koba \\ Institutional Area, Gandhinagar-382007, Gujarat (INDIA) \\ *Corresponding author: E-mail: jeenen@gmail.com \\ Phone number: (+912692) 226856*218
}

Received: 03-30-2020

\begin{abstract}
The neutral rhenium(I) complexes (I-VI) of type $\left[\operatorname{ReCl}(\mathrm{CO})_{3} \mathrm{~L}^{\mathrm{n}}\right]$ \{where $\mathrm{L}^{1}=7$-phenyl-5-(pyridin-2-yl)pyrazolo $[1,5$-a $]$ pyrimidine, $\mathrm{L}^{2}=7$-(4-bromophenyl)-5-(pyridin-2-yl)pyrazolo[1,5-a]pyrimi- dine, $\mathrm{L}^{3}=7$-(4-chlorophenyl)-5-(pyridin-2-yl)pyrazolo[1,5-a]pyrimidine, $\quad \mathrm{L}^{4}=7$-(2-chlorophenyl) -5 -(pyridin-2-yl)pyrazolo[1,5-a]pyrimidine, $\quad \mathrm{L}^{5}=$ 7-(4-methoxyphenyl)-5-(pyridin-2-yl)pyrazolo [1,5-a]pyrimidine, $\mathrm{L}^{6}=5$-(pyridin-2-yl)-7-(p-tolyl)pyrazolo[1,5-a]pyrimidine were synthesized and characterized by ${ }^{13} \mathrm{C}-\mathrm{APT},{ }^{1} \mathrm{H}-\mathrm{NMR}$, IR, electronic spectra, magnetic moment and conductance measurement. The anti-proliferative activity on HCT116 cells by MTT assay suggests potent cytotoxic nature of complexes, some complexes even have better activity than standard drug cisplatin, oxaliplatin, and carboplatin. The complexes were found to have better antimicrobial activity compare to pyrazolo pyrimidine ligands. The theoretical study of compounds-DNA interactions was examined by molecular docking as a supportive tool to the experimental data, which suggests the groove mode of binding. The values of docking energy for compounds-DNA interaction were found in the range of -230.31 to $-288.34 \mathrm{~kJ} / \mathrm{mol}$. The intrinsic binding constant values of complexes $\left(1.1-3.5 \times 10^{5} \mathrm{M}^{-1}\right)$ were found higher than the ligands $\left(0.32-1.8 \times 10^{5} \mathrm{M}^{-1}\right)$.
\end{abstract}

Keywords: In vitro cytotoxicity; Molecular modelling; Anti-proliferative activity; Groove binding

\section{Introduction}

Metal carbonyl moieties, such as $\left\{\mathrm{M}(\mathrm{CO})_{3}\right\}(\mathrm{M}=\mathrm{Cr}$, $\mathrm{Mn}, \mathrm{Re}, \mathrm{Fe})$, can attach to the biomolecules capable of molecular recognition, to label and assay, specific biological receptors. When $\mathrm{M}=\mathrm{Tc}$ or $\mathrm{Re}$, the same idea is used to introduce radioactive ${ }^{99 \mathrm{~m}} \mathrm{Tc},{ }^{186} \mathrm{Re}$, or ${ }^{188} \mathrm{Re}$ at a receptor for radiopharmaceutical applications. ${ }^{1,2}$ There has been considerable interest in testing metal carbonyls for anticancer activity. ${ }^{3}$ For example, $\left[\mathrm{Co}_{2}(\mathrm{CO})_{6}\left(\mathrm{HC}_{2} \mathrm{C}-\mathrm{CH}_{2} \mathrm{O}_{2} \mathrm{CC}_{6} \mathrm{H}_{4}-2\right.\right.$ $\mathrm{OH})$ ] is more active than cisplatin on the human mammary tumor cell lines MCF-7 and MDA-MB-231. ${ }^{4}$ Also $\left[\left\{\eta^{5}-\right.\right.$ $\left.\left(4-\mathrm{Me}_{2} \mathrm{~N}\left\{\mathrm{CH}_{2}\right\}_{4} \mathrm{OC}_{6} \mathrm{H}_{4}\right)-\left(4-\mathrm{HOC}_{6} \mathrm{H}_{4}\right) \mathrm{CHCHEtC}_{5} \mathrm{H}_{4}\right\}$ $\operatorname{Re}(\mathrm{CO})_{3}$ ] has been shown to behave similarly to tamoxifen, and it appears that the observed antiproliferative effect is dependent on the oestradiol receptor $a^{5}$<smiles>c1cnc2ccnn2c1</smiles>

pyrazolo $[1,5-a]$ pyrimidine

Pyrazole and pyrimidine derivatives attracted organic chemists very much due to their biological and chemotherapeutic importance. Pyrazolo pyrimidines and related fused heterocycles are of interest as potential bioactive molecules. They are known to exhibit pharmacological ac- 
tivities such as CNS depressant, ${ }^{6}$ neuroleptic, ${ }^{7}$ and tuberculostatic. ${ }^{8}$ Recently, the chemistry of pyrazolo[1,5-a]pyrimidines attracted great attention as a synthetically important class of compounds. ${ }^{9}$ They represent biologically important compounds of purine analogues and this class has attracted wide pharmaceutical interest as inhibitors of lymphocyte-specific kinase (Lck) with enzymatic, cellular, and in vivo potency. ${ }^{10}$ In 2003, a research group from NRC synthesized some pyrazolo[1,5-a]pyrimidines and studied their biological effects as an anti-inflammatory, analgesic, and antipyretic drugs in comparison to novalgin..$^{11}$ The choice of the ligand is very important for the development of new radiopharmaceuticals reagents; thus, studies on rhenium(I) complexes with ligands as aromatic $\mathrm{N}$-heterocycles have shown a great effectiveness. ${ }^{12}$

In continuation of our earlier work, ${ }^{13}$ the present study illustrates the synthesis of new heterocyclic ligands and their organometallic rhenium complexes. Heterocyclic compounds have significant biological importance upon chelation with pentacarbonyl chloro rhenium(I) and presence of carbonyls group attached with metal which further enhanced the biological activity.

\section{Experimental}

Materials and methods: All the chemicals and solvents were of reagent grade, 2-acetyl thiophene, substituted aldehyde were purchased from Merck Limited (India), different substituted phenyl hydrazine were purchased from Thirumalai Chemicals Ltd. (TCL), potassium-tert-butoxide, potassium hydroxide purchased from Sisco Research Laboratories Pvt. Ltd. (SRL), pentacarbonyl chloro rhenium(I) purchased from Sigma Aldrich (USA). Luria broth and nutrient broth were purchased from Himedia (India). Agarose and Luria Broth (LB) were purchased from Hi-media Laboratories Pvt. Ltd., India. Culture of two Gram(+ve), i.e. Staphylococcus aureus (S. aureus) (MTCC3160) and Bacillus subtilis (MTCC-7193), and three Gram(-ve), i.e. Serratia marcescens (MTCC-7103), Pseudomonas aeruginosa (MTCC-1688) and Escherichia coli (MTCC-433), were purchased from Institute of Microbial Technology (Chandigarh, India). S. cerevices Var. Paul Linder 3360 was obtained from IMTECH, Chandigarh, India. HS DNA was purchased from Sigma Aldrich Chemical Co. (India). Human colorectal carcinoma (HCT 116) cells were obtained from the cell repository, National Center for Cell Science (NCCS), Pune, Maharashtra, India.

Physical measurements: The ${ }^{1} \mathrm{H}$ and ${ }^{13} \mathrm{C}$ NMR spectra were recorded on a Bruker Avance $(400 \mathrm{MHz})$. Infrared spectra were recorded on an FT-IR ABB Bomen MB 3000 spectrophotometer in the range $4000-400 \mathrm{~cm}^{-1}$. C, $\mathrm{H}$, and $\mathrm{N}$ elemental analyses were performed with a Heraeus, Germany CHNO RAPID. Molar conductance was meas- ured using a conductivity meter model no. EQ-660A, Mumbai (India). Melting points $\left({ }^{\circ} \mathrm{C}\right.$, uncorrected) were determined in open capillaries on the ThermoCal10 melting point apparatus (Analab Scientific Pvt. Ltd, India). The electronic spectra were recorded on a UV-160A UV-Vis spectrophotometer, Shimadzu (Japan). The minimum inhibitory concentration (MIC) study was carried out using laminar airflow cabinet (Toshiba, Delhi, India). Hydrodynamic chain length study was carried out by a viscometric measurement bath. Photo quantization of the gel after electrophoresis was carried out on AlphaDigiDocTM RT. Version V.4.0.0 PC-Image software.

General method for synthesis of pyrazolo[1,5-a]pyrimidines ligands $\left(\mathbf{L}^{1}-\mathbf{L}^{6}\right)$ : The $\alpha, \beta$ unsaturated carbonyl compounds (3a-3f) were synthesized using literature procedure. ${ }^{14}$ Syntheses of the pyrazolo[1,5-a]pyrimidines based ligands $\left(\mathrm{L}^{1}-\mathrm{L}^{6}\right)$ were carried out using Lipson and coworkers method. ${ }^{15}$ To a solution of the $\alpha, \beta$-unsaturated carbonyl compounds (3a-3f) $(\sim 2.391 \mathrm{mmol})$ in $10 \mathrm{~mL}$ of DMF, 1H-pyrazol-3-amine (4a) ( 198.7 mg, 2.391 $\mathrm{mmol})$ and $\mathrm{KOH}(\sim 15 \mathrm{mg}, \sim 2.391 \mathrm{mmol})$ solution were added. The reaction mixture was refluxed for $30 \mathrm{~min}$. Completion of the reaction was checked by TLC plates, the excess of solvent was removed under reduced pressure and the reaction mixture was cooled on an ice bath. The reaction mixture was extracted with ethyl acetate $(20 \mathrm{~mL} \times 2)$ and washed thoroughly with water $(25 \mathrm{~mL} \times 2)$. The brine solution of sodium chloride was added to it and dried over sodium sulphate. The resulting mixture was concentrated under vacuum to obtain pyrazolo[1,5-a]pyrimidine based ligands as products. The ${ }^{1} \mathrm{H}$ and ${ }^{13} \mathrm{C}$ NMR spectra are shown in supplementary material 1 and 2 respectively.

Synthesis of 7-phenyl-5-(pyridin-2-yl)pyrazolo[1,5-a] pyrimidine $\left(L^{1}\right)$ : The ligand $\left(\mathrm{L}^{1}\right)$ was prepared by using enone (3a) (500 mg, $2.391 \mathrm{mmol})$ and $1 \mathrm{H}$-pyrazole-3amine (4a) (198.7 mg, $2.391 \mathrm{mmol})$. Yield: 84.2\%; Color: yellowish amorphous solid; $\mathbf{m p} 170{ }^{\circ} \mathrm{C}$; Mol. wt.: $272.31 \mathrm{~g} /$ mol; Empirical formula: $\mathrm{C}_{17} \mathrm{H}_{12} \mathrm{~N}_{4}$, Elemental analysis: Calc. (\%): C, 74.98; H, 4.44; N, 20.58; found. C, 74.88; H, 4.40; N, 20.58; Mass spectra (m/z \%): $272.20(100)\left[\mathrm{M}^{+}\right]$;

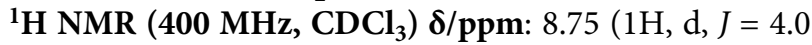
$\left.\mathrm{Hz}, \mathrm{H}_{6^{\prime \prime}}\right), 8.59\left(1 \mathrm{H}, \mathrm{d}, J=8.0 \mathrm{~Hz}, \mathrm{H}_{4}{ }^{\prime \prime}\right), 8.22\left(1 \mathrm{H}, \mathrm{s}, \mathrm{H}_{7}\right), 8.16$ $\left(2 \mathrm{H}, \mathrm{dd}, J=4.4 \mathrm{~Hz}, J=3.2 \mathrm{~Hz}, \mathrm{H}_{3^{\prime \prime}} 5^{\prime \prime}\right), 7.89(2 \mathrm{H}, \mathrm{d}, J=1.6$ $\left.\mathrm{Hz}, \mathrm{H}_{2,6}{ }^{\prime}\right), 7.60\left(1 \mathrm{H}, \mathrm{d}, J=3.6 \mathrm{~Hz}, \mathrm{H}_{3}\right), 7.41\left(3 \mathrm{H}, \mathrm{m}, \mathrm{H}_{3,}, 4^{\prime}\right.$, 5), $6.86\left(1 \mathrm{H}, \mathrm{d}, J=2.4 \mathrm{~Hz}, \mathrm{H}_{4}\right) .{ }^{13} \mathrm{C}$ NMR $(100 \mathrm{MHz}$,

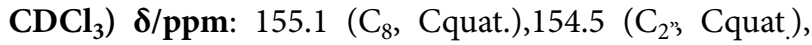
$149.8\left(\mathrm{C}_{6}\right.$, Cquat $), 149.2\left(\mathrm{C}_{6}, \mathrm{CH}\right), 146.9\left(\mathrm{C}_{5 \mathrm{a}}\right.$, Cquat $)$, $145.2\left(\mathrm{C}_{4}\right.$,, $\left.\mathrm{CH}\right), 136.4\left(\mathrm{C}_{3},-\mathrm{CH}\right), 131.6\left(\mathrm{C}_{1}\right.$, Cquat. $) 130.9$ $\left(\mathrm{C}_{3,5},-\mathrm{CH}\right), 129.4\left(\mathrm{C}_{4},-\mathrm{CH}\right), 128.6\left(\mathrm{C}_{2}, 6,-\mathrm{CH}\right), 124.8$ $\left(\mathrm{C}_{5},-\mathrm{CH}\right), 121.6\left(\mathrm{C}_{3},-\mathrm{CH}\right), 105.2\left(\mathrm{C}_{7},-\mathrm{CH}\right), 97.5\left(\mathrm{C}_{4}\right.$, $-\mathrm{CH}$ ). [Total signal observed $=15$ : signal of $\mathrm{C}=5$ (phenyl ring- $\mathrm{C}=1$, pyrazolo[1,5-a]pyrimidine- $\mathrm{C}=3$, pyridine ring- $\mathrm{C}=1$ ), signal of $\mathrm{CH}=10$ (pyrazolo[1,5-a]pyrimidine- $\mathrm{CH}=3$, phenylring- $\mathrm{CH}=3$, pyridine ring- $\mathrm{CH}=4)$ ]; IR (KBr, 4000-400 cm$\left.^{-1}\right): 2930 v(=\mathrm{C}-\mathrm{H})$ ar., $1551 v(\mathrm{C}=\mathrm{N})$, 
$1504(\mathrm{C}-\mathrm{H})$ bending, $1251 v(\mathrm{C}-\mathrm{N}), 1597 v(\mathrm{C}=\mathrm{C})$ conjugated alkenes, $763 v($ Ar-H) adjacent hydrogen.

7-(4-Bromophenyl)-5-(pyridin-2-yl)pyrazolo[1,5-a] pyrimidine $\left(L^{2}\right)$ : The ligand $\left(\mathrm{L}^{2}\right)$ was prepared by using enone (3b) (500 mg, $1.730 \mathrm{mmol}$ ) and $1 \mathrm{H}$-pyrazole-3amine (4a) (143.8 mg, $1.730 \mathrm{mmol}$ ). Yield: $84.2 \%$; Color: yellowish amorphous solid; $\mathbf{m p ~} 182^{\circ} \mathrm{C}$; Mol. wt.: $351.21 \mathrm{~g} /$ mol; Empirical formula: $\mathrm{C}_{17} \mathrm{H}_{11} \mathrm{BrN}_{4}$, Elemental analysis: Calc. (found) (\%): C, 58.14; H, 3.16; N, 15.95; found. C, 58.08; H, 3.11; N, 15.90; Mass spectra (m/z \%): 350.4 (100) $\left[\mathrm{M}^{+}\right], 352.4[\mathrm{M}+2] ;{ }^{1} \mathbf{H}$ NMR $\left(400 \mathrm{MHz}, \mathbf{C D C l}_{3}\right) \boldsymbol{\delta} /$ ppm: $8.75\left(1 \mathrm{H}, \mathrm{d}, J=4.4 \mathrm{~Hz}, \mathrm{H}_{6^{\prime \prime}}\right), 8.59\left(1 \mathrm{H}, \mathrm{d}, J=8.0, \mathrm{H}_{4^{\prime \prime}}\right)$, $8.21\left(1 \mathrm{H}, \mathrm{s}, \mathrm{H}_{7}\right), 8.14\left(2 \mathrm{H}, \mathrm{dd}, J=3.2 \mathrm{~Hz}, 2 \mathrm{~Hz}, \mathrm{H}_{3^{\prime \prime}} 5^{\prime \prime}\right), 7.94$ $\left(1 \mathrm{H}, \mathrm{d}, J=6.4 \mathrm{~Hz}, \mathrm{H}_{6}\right), 7.91\left(1 \mathrm{H}, \mathrm{d}, J=7.6 \mathrm{~Hz}, \mathrm{H}_{2^{\prime}}\right), 7.76$ $\left(2 \mathrm{H}, \mathrm{d}, J=2.0 \mathrm{~Hz}, \mathrm{H}_{3^{\prime}, \mathrm{H} 5^{\prime}}\right), 7.43(1 \mathrm{H}, \mathrm{dd}, J=8.0 \mathrm{~Hz}, 1.6 \mathrm{~Hz}$, $\left.\mathrm{H}_{3}\right), 6.87\left(1 \mathrm{H}, \mathrm{d}, J=1.2 \mathrm{~Hz}, \mathrm{H}_{4}\right) \cdot{ }^{13} \mathrm{C}$ NMR $(100 \mathrm{MHz}$,

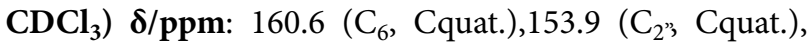
153.1 ( $\mathrm{C}_{5 \mathrm{a}}$, Cquat.), 148.8 ( $\mathrm{C}_{8}$, Cquat. $), 148.9\left(\mathrm{C}_{6},-\right.$ $\mathrm{CH}), 145.7\left(\mathrm{C}_{1}\right.$, Cquat. $), 145.2\left(\mathrm{C}_{4},-\mathrm{CH}\right), 137.9\left(\mathrm{C}_{3},-\right.$ $\mathrm{CH}), 130.9\left(\mathrm{C}_{3,5},-\mathrm{CH}\right), 125.5\left(\mathrm{C}_{2,6},-\mathrm{CH}\right), 122.3\left(\mathrm{C}_{4}\right.$, Cquat.), $121.1\left(\mathrm{C}_{5},-\mathrm{CH}\right), 117.6\left(\mathrm{C}_{3}\right.$, $\left.-\mathrm{CH}\right), 103.3\left(\mathrm{C}_{7},-\right.$ $\mathrm{CH}), 97.9\left(\mathrm{C}_{4},-\mathrm{CH}\right)$. [Total signal observed $=15$ : signal of $\mathrm{C}=6$ ( $p$-Br-phenyl ring- $\mathrm{C}=2$, pyrazolo[1,5-a]pyrimidine- $\mathrm{C}=3$, pyridine ring- $\mathrm{C}=1$ ), signal of $\mathrm{CH}=9$ (pyrazolo[1,5-a]pyrimidine- $\mathrm{CH}=3, p-\mathrm{Br}$ phenyl ring- $\mathrm{CH}=2$, pyridine ring- $\mathrm{CH}=4)$ ]; IR $\left(\mathbf{K B r}, \mathbf{4 0 0 0}-\mathbf{4 0 0} \mathbf{~ c m}^{-1}\right): 2925$ $v(=\mathrm{C}-\mathrm{H})$ ar., $1558 v(\mathrm{C}=\mathrm{N}), 1490(\mathrm{C}-\mathrm{H})$ bending, 1204 $v(\mathrm{C}-\mathrm{N}), 1597 v(\mathrm{C}=\mathrm{C})$ conjugated alkenes, $764 v(\mathrm{Ar}-\mathrm{H})$ adjacent hydrogen.

7-(4-Chlorophenyl)-5-(pyridin-2-yl)pyrazolo[1,5-a] pyrimidine $\left(L^{3}\right)$ : The ligand $\left(\mathrm{L}^{3}\right)$ was prepared by using enone (3c) (500 mg, $2.044 \mathrm{mmol})$ and $1 \mathrm{H}$-pyrazole-3amine (4a) (169.8 mg, $2.044 \mathrm{mmol})$. Yield: 85.4\%; Color: yellowish amorphous solid; $\mathbf{m p ~} 178{ }^{\circ} \mathrm{C}$; Mol. wt.: $306.75 \mathrm{~g} /$ mol; Empirical formula: $\mathrm{C}_{17} \mathrm{H}_{11} \mathrm{ClN}_{4}$, Calc. (\%): C, 66.56; H, 3.61; N, 18.26; found. C, 66.55; H, 3.58; N, 18.24; Mass spectra (m/z \%): $306.20(100)\left[\mathrm{M}^{+}\right], 308.20[\mathrm{M}+2] ;{ }^{1} \mathbf{H}$

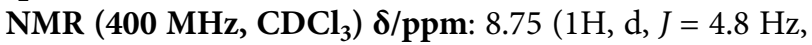
$\left.\mathrm{H}_{6^{\prime \prime}}\right), 8.59\left(1 \mathrm{H}, \mathrm{d}, J=8.0 \mathrm{~Hz}, \mathrm{H}_{4^{\prime \prime}}\right), 8.22\left(1 \mathrm{H}, \mathrm{s}, \mathrm{H}_{7}\right), 8.17$ $\left.\left(2 \mathrm{H}, \mathrm{dd}, J=8.4,4.0 \mathrm{~Hz}, \mathrm{H}_{3^{\prime \prime}, 5}\right), 7.93\left(2 \mathrm{H}, \mathrm{d}, J=2.0 \mathrm{~Hz}, \mathrm{H}_{2,6}\right)^{\prime}\right)$, $7.58\left(1 \mathrm{H}, \mathrm{d}, J=8.4 \mathrm{~Hz}, \mathrm{H}_{3}\right), 7.44\left(2 \mathrm{H}, \mathrm{d}, J=4.0 \mathrm{~Hz}, \mathrm{H}_{3,5^{\prime}}\right)$, $6.87\left(1 \mathrm{H}, \mathrm{d}, J=2.4 \mathrm{~Hz}, \mathrm{H}_{4}\right) .{ }^{13} \mathrm{C}$ NMR $\left(100 \mathrm{MHz}, \mathrm{CDCl}_{3}\right)$

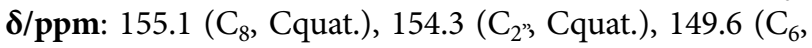
Cquat.), $149.2\left(\mathrm{C}_{6,},-\mathrm{CH}\right), 145.7\left(\mathrm{C}_{4},-\mathrm{CH}\right), 145.2\left(\mathrm{C}_{4}\right.$, Cquat.), $137.2\left(\mathrm{C}_{3},-\mathrm{CH}\right), 130.8$ ( $\mathrm{C}_{5 \mathrm{a}}$, Cquat.), $129.9\left(\mathrm{C}_{3,5}\right.$, $-\mathrm{CH}), 129.4$ ( $\mathrm{C}_{1}$, Cquat.), $129.0\left(\mathrm{C}_{2}, 6,-\mathrm{CH}\right), 124.9\left(\mathrm{C}_{5}\right.$, $\mathrm{CH}), 121.7\left(\mathrm{C}_{3^{\prime \prime}}, \mathrm{CH}\right), 104.9\left(\mathrm{C}_{7},-\mathrm{CH}\right), 97.68\left(\mathrm{C}_{4},-\mathrm{CH}\right)$. [Total signal observed $=15$ : signal of $\mathrm{C}=6$ ( $p$-Cl-phenyl ring- $\mathrm{C}=2$, pyrazolo[1,5-a]pyrimidine- $\mathrm{C}=3$, pyridine ring- $\mathrm{C}=1$ ), signal of $\mathrm{CH}=9$ (pyrazolo[1,5-a]pyrimidine$\mathrm{CH}=3$, $p$-Cl-phenyl ring- $\mathrm{CH}=2$, pyridine ring- $\mathrm{CH}=4)$ ]; IR (KBr, 4000-400 $\left.\mathbf{c m}^{-1}\right)$ : $2922 v(=\mathrm{C}-\mathrm{H})$ ar., $1551 v(\mathrm{C}=\mathrm{N})$, $1504(\mathrm{C}-\mathrm{H})$ bending, $1190 v(\mathrm{C}-\mathrm{N}), 1605 v(\mathrm{C}=\mathrm{C})$ conjugated alkenes, $756 v($ Ar-H) adjacent hydrogen.

7-(2-Chlorophenyl)-5-(pyridin-2-yl)pyrazolo[1,5-a] pyrimidine $\left(L^{4}\right)$ : This ligand $\left(\mathrm{L}^{1}\right)$ was prepared by using enone (3d) (500 mg, $2.044 \mathrm{mmol})$ and 1H-pyrazole-3amine (4a) (169.8 mg, $2.044 \mathrm{mmol}$ ). Yield: 79.5\%; Color: yellowish amorphous solid; $\mathbf{m p} 180^{\circ} \mathrm{C}$; Mol. wt.: $306.75 \mathrm{~g} /$ mol; Empirical formula: $\mathrm{C}_{17} \mathrm{H}_{11} \mathrm{ClN}_{4}$, Calc. (found) (\%): C, 66.56; H, 3.61; N, 18.26; found. C, 66.50; H, 3.60; N, 18.23; Mass spectra (m/z \%): 306.82 (100) [M+], 308.82

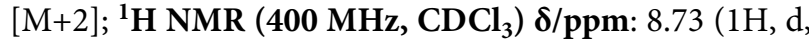
$\left.J=3.6 \mathrm{~Hz}, \mathrm{H}_{6^{\prime \prime}}\right), 8.61\left(1 \mathrm{H}, \mathrm{d}, J=8.0 \mathrm{~Hz}, \mathrm{H}_{4^{\prime \prime}}\right), 8.19(1 \mathrm{H}, \mathrm{d}, J$ $\left.=2.4 \mathrm{~Hz}, \mathrm{H}_{5^{\prime \prime}}\right), 8.09\left(1 \mathrm{H}, \mathrm{s}, \mathrm{H}_{7}\right), 7.93(1 \mathrm{H}, \mathrm{d}, J=1.6 \mathrm{~Hz}$, $\left.\mathrm{H}_{3^{\prime \prime}}\right), 7.62\left(2 \mathrm{H}, \mathrm{m}, \mathrm{H}_{4,5^{\prime}}\right), 7.51\left(2 \mathrm{H}, \mathrm{m}, \mathrm{H}_{3,6^{\prime}}\right), 7.41(1 \mathrm{H}, \mathrm{d}, J$ $\left.=5.2 \mathrm{~Hz}, \mathrm{H}_{3}\right), 6.88\left(1 \mathrm{H}, \mathrm{d}, J=2.4 \mathrm{~Hz}, \mathrm{H}_{4}\right) \cdot{ }^{13} \mathrm{C}$ NMR $(100$

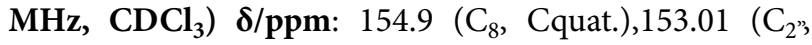
Cquat.), $149.28\left(\mathrm{C}_{6}\right.$, $\left.-\mathrm{CH}\right), 148.9$ ( $\mathrm{C}_{6}$, Cquat.), 145.5 ( $\mathrm{C}_{4}$, $-\mathrm{CH}), 145.1\left(\mathrm{C}_{5 \mathrm{a}}\right.$, Cquat.), $137.10\left(\mathrm{C}_{3},-\mathrm{CH}\right), 133.7\left(\mathrm{C}_{2}\right.$, Cquat.), $131.57\left(\mathrm{C}_{3},-\mathrm{CH}\right), 131.1\left(\mathrm{C}_{5},-\mathrm{CH}\right), 130.2\left(\mathrm{C}_{4},-\right.$ $\mathrm{CH}), 128.6\left(\mathrm{C}_{1}\right.$, Cquat. $), 127.1\left(\mathrm{C}_{6},-\mathrm{CH}\right), 124.9\left(\mathrm{C}_{5}\right.$, $\mathrm{CH}), 121.8\left(\mathrm{C}_{3},-\mathrm{CH}\right), 105.2\left(\mathrm{C}_{7},-\mathrm{CH}\right), 97.72\left(\mathrm{C}_{4},-\mathrm{CH}\right)$. [Total signal observed $=17$ : signal of $\mathrm{C}=6(\mathrm{o}-\mathrm{Cl}$-phenyl ring- $\mathrm{C}=2$, pyrazolo[1,5-a]pyrimidine- $\mathrm{C}=3$, phenyl ring- $\mathrm{C}=1$ ), signal of $\mathrm{CH}=11$ (pyrazolo[1,5-a]pyrimidine- $\mathrm{CH}=3, o-\mathrm{Cl}$ phenylring- $\mathrm{CH}=4$, pyridine ring- $\mathrm{CH}=$ 4)]; IR (KBr, 4000-400 $\mathbf{~ c m}^{-1}$ ): $2922 v(=\mathrm{C}-\mathrm{H})$ ar., 1551 $v(\mathrm{C}=\mathrm{N}), 1504 v(\mathrm{C}-\mathrm{H})$ bending, $1190 v(\mathrm{C}-\mathrm{N}), 1605 v(\mathrm{C}=\mathrm{C})$ conjugated alkenes, $758 v($ Ar- $\mathrm{H})$ adjacent hydrogen.

7-(4-Methoxyphenyl)-5-(pyridin-2-yl)pyrazolo[1,5-a]pyrimidine $\left(L^{5}\right)$ : The ligand $\left(\mathrm{L}^{5}\right)$ was prepared by using enone (3e) $(500 \mathrm{mg}, 2.082 \mathrm{mmol})$ and $1 \mathrm{H}$-pyrazole-3-amine (4a) (173 mg, $2.082 \mathrm{mmol}$ ). Yield: 87.6\%; Color: yellowish amorphous solid; $\mathbf{m p} 178{ }^{\circ} \mathrm{C}$; Mol. wt.: $302.34 \mathrm{~g} / \mathrm{mol}$; Empirical formula: $\mathrm{C}_{18} \mathrm{H}_{14} \mathrm{~N}_{4} \mathrm{O}$, Calc. (found) (\%): C, 71.51; H, 4.67; N, 18.53; found. C, 71.48; $\mathrm{H}, 4.62 ; \mathrm{N}, 18.56$; Mass spectra (m/z \%): 302.20 (100)

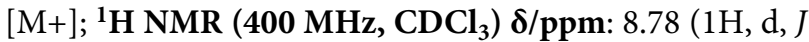
$\left.=4.4 \mathrm{~Hz}, \mathrm{H}_{6^{\prime \prime}}\right), 8.51\left(1 \mathrm{H}, \mathrm{d}, J=8.0 \mathrm{~Hz}, \mathrm{H}_{4^{\prime \prime}}\right), 8.33(1 \mathrm{H}, \mathrm{s}, \mathrm{Hz}$,

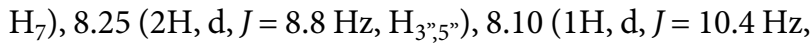
$\left.\mathrm{H}_{6}{ }^{\prime}\right), 8.03\left(1 \mathrm{H}, \mathrm{d}, J=7.6 \mathrm{~Hz}, \mathrm{H}_{2}{ }^{2}\right), 7.58(1 \mathrm{H}, \mathrm{d}, J=5.2 \mathrm{~Hz}$, $\left.\mathrm{H}_{3}\right), 7.19\left(2 \mathrm{H}, \mathrm{d}, J=8.8 \mathrm{~Hz}, \mathrm{H}_{3,5}{ }^{2}\right), 6.92(1 \mathrm{H}, \mathrm{d}, J=2.0 \mathrm{~Hz}$, $\left.\mathrm{H}_{4}\right), 3.09\left(3 \mathrm{H}, \mathrm{s},-\mathrm{OCH}_{3}\right) .{ }^{13} \mathrm{C} \mathrm{NMR}\left(\mathbf{1 0 0 ~ M H z}, \mathbf{C D C l}_{3}\right) \boldsymbol{\delta} /$ ppm: 161.9 ( $\mathrm{C}_{4}$, Cquat.), 154.7 ( $\mathrm{C}_{8}$, Cquat.), $154.01\left(\mathrm{C}_{2}\right.$, Cquat.), $149.9\left(\mathrm{C}_{6}\right.$, $\left.-\mathrm{CH}\right), 149.7\left(\mathrm{C}_{6}\right.$, Cquat. $), 146.7\left(\mathrm{C}_{4}\right.$, $\mathrm{CH}), 146.3$ ( $\mathrm{C}_{5 \mathrm{a}}$, Cquat.), $138.1\left(\mathrm{C}_{3},-\mathrm{CH}\right), 131.7\left(\mathrm{C}_{2^{\prime}, 6}\right.$, $\mathrm{CH}), 125.8\left(\mathrm{C}_{5}\right.$, $\left.-\mathrm{CH}\right), 123.4\left(\mathrm{C}_{1}\right.$, Cquat. $), 121.5\left(\mathrm{C}_{3, \text {, }}\right.$ $\mathrm{CH}), 114.5\left(\mathrm{C}_{7},-\mathrm{CH}\right), 103.8\left(\mathrm{C}_{3,5},-\mathrm{CH}\right), 97.6\left(\mathrm{C}_{4},-\mathrm{CH}\right)$, $55.9\left(-\mathrm{OCH}_{3}\right)$. [Total signal observed $=16$ : signal of $\mathrm{C}=$ $6\left(p-\mathrm{OCH}_{3}\right.$-phenyl ring- $\mathrm{C}=2$, pyrazolo[ $\left.1,5-\mathrm{a}\right]$ pyrimidine- $\mathrm{C}=3$, pyridine ring- $\mathrm{C}=1$ ), signal of $\mathrm{CH}=9$ (pyrazolo[1,5-a]pyrimidine- $\mathrm{CH}=3, p-\mathrm{OCH}_{3}$ phenylring- $\mathrm{CH}=$ 2 , pyridine ring- $\left.\mathrm{CH}=4),-\mathrm{OCH}_{3}=1\right] ; \mathbf{I R}(\mathbf{K B r}, \mathbf{4 0 0 0}-\mathbf{4 0 0}$ cm $\left.^{-1}\right)$ : $2922 v(=\mathrm{C}-\mathrm{H})$ ar., $1551 v(\mathrm{C}=\mathrm{N}), 1514(\mathrm{C}-\mathrm{H})$ bending, $1188 v(\mathrm{C}-\mathrm{N}), 1597 v(\mathrm{C}=\mathrm{C})$ conjugated alkenes, 764 $v($ Ar-H) adjacent hydrogen.

5-(Pyridin-2-yl)-7-(p-tolyl)pyrazolo[1,5-a]pyrimidine $\left(L^{6}\right)$ : The ligand $\left(L^{6}\right)$ was prepared by using enone $(3 f)$ (500 $\mathrm{mg}, 2.231 \mathrm{mmol}$ ) and $1 \mathrm{H}$-pyrazole-3-amine (4a) (185.4 mg, $2.231 \mathrm{mmol})$. Yield: 82.5\%; Color: yellowish amorphous solid; $\mathbf{m p} 175^{\circ} \mathrm{C}$; Mol. wt.: $286.34 \mathrm{~g} / \mathrm{mol}$; Em-

Varma et al.: Synthesis, Characterization and Biological Application ... 
pirical formula: $\mathrm{C}_{18} \mathrm{H}_{14} \mathrm{~N}_{4}$, Calc. (found) (\%): C, 75.50; $\mathrm{H}$, 4.93; N, 19.57; found. C, 75.46; H, 4.90; N, 19.55; Mass spectra (m/z \%): $286.60(100)\left[\mathrm{M}^{+}\right] ;{ }^{1} \mathrm{H}$ NMR (400 MHz,

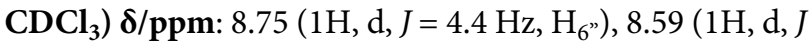
$\left.=8.0 \mathrm{~Hz}, \mathrm{H}_{4}\right), 8.23\left(1 \mathrm{H}, \mathrm{s}, \mathrm{H}_{7}\right), 8.22(2 \mathrm{H}, \mathrm{dd}, J=2.4 \mathrm{~Hz}, 1.6$ $\left.\mathrm{Hz}, \mathrm{H}_{3^{\prime}}, 5^{\prime \prime}\right), 8.08\left(2 \mathrm{H}, \mathrm{dd}, J=10.0 \mathrm{~Hz}, 8.0 \mathrm{~Hz}, \mathrm{H}_{2,6^{\prime}}\right), 7.41$ $\left(3 \mathrm{H}, \mathrm{d}, J=7.6 \mathrm{~Hz}, \mathrm{H}_{3,3}, 5^{\prime}\right), 6.85\left(1 \mathrm{H}, \mathrm{d}, J=2.0 \mathrm{~Hz}, \mathrm{H}_{4}\right), 2.49$

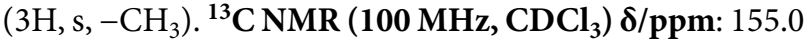
( $\mathrm{C}_{8}$, Cquat.),154.6 ( $\mathrm{C}_{2}$, Cquat.), 149.7 (C ${ }_{6}$, Cquat.), 149.2 $\left(\mathrm{C}_{6}\right.$, $\left.-\mathrm{CH}\right), 147.07$ ( $\mathrm{C}_{5 \mathrm{a}}$, Cquat. $), 145.1\left(\mathrm{C}_{4},-\mathrm{CH}\right), 141.3$ ( $\mathrm{C}_{4}$, Cquat.), $137.0\left(\mathrm{C}_{3},-\mathrm{CH}\right), 129.3\left(\mathrm{C}_{3}, 5,-\mathrm{CH}\right), 129.2$ ( $\mathrm{C}_{1}$, Cquat. $), 128.6\left(\mathrm{C}_{2}, 6,-\mathrm{CH}\right), 124.7\left(\mathrm{C}_{5}\right.$,,$\left.-\mathrm{CH}\right), 121.6$ $\left(\mathrm{C}_{3}\right.$, $\left.-\mathrm{CH}\right), 104.73\left(\mathrm{C}_{7},-\mathrm{CH}\right), 97.4\left(\mathrm{C}_{4},-\mathrm{CH}\right), 21.5$ ($\left.\mathrm{CH}_{3}\right)$. [Total signal observed $=16$ : signal of $\mathrm{C}=6\left(p-\mathrm{CH}_{3}\right.$ phenyl ring- $\mathrm{C}=2$, pyrazolo $[1,5-\mathrm{a}]$ pyrimidine- $\mathrm{C}=3$, pyridine ring- $\mathrm{C}=1$ ), signal of $\mathrm{CH}=9$ (pyrazolo[1,5-a]pyrimidine- $\mathrm{CH}=3, p-\mathrm{CH}_{3}$-phenylring- $\mathrm{CH}=2$, pyridine ring$\mathrm{CH}=4),-\mathrm{CH}_{3}=1$ ]; IR (KBr, $\left.4000-400 \mathbf{~ c m}^{-1}\right): 2923$ $v(=\mathrm{C}-\mathrm{H})$ ar., $1551 \quad v(\mathrm{C}=\mathrm{N}), 1512(\mathrm{C}-\mathrm{H})$ bending, 1196 $v(\mathrm{C}-\mathrm{N}), 1597 v(\mathrm{C}=\mathrm{C})$ conjugated alkenes, $764 v(\mathrm{Ar}-\mathrm{H})$ adjacent hydrogen.
General synthesis of complexes: The metal carbonyl complexes (I-VI) were synthesized using pentacarbonyl chloro rhenium(I) and ligands $\left(\mathrm{L}^{1}-\mathrm{L}^{6}\right)$ in ethanol in a $1: 1$ proportion. ${ }^{16}$

Synthesis of $\left[\mathrm{Re}(\mathrm{CO})_{3}\left(\mathrm{~L}^{1}\right) \mathrm{Cl}\right](\mathrm{I})$ : Ethanolic solution of the precursor of $\left[\mathrm{Re}(\mathrm{CO})_{5} \mathrm{Cl}\right](100 \mathrm{mg}, 0.276 \mathrm{mmol})$ was refluxed for 10 minutes. Then a solution of ligand $\left(\mathrm{L}^{1}\right)$ (75 mg, $0.276 \mathrm{mmol}$ in $10 \mathrm{~mL}$ ethanol), was added and the reaction was stirred yielding a solution. The resulting mixture was stirred at $60{ }^{\circ} \mathrm{C}$ for $5-6 \mathrm{hr}$. Progress of reaction was monitored by TLC after completion of reaction the solution was filtered through celite in order to remove solid particles and the solvent was removed under reduced pressure the orange red product was obtained. The proposed reaction for the synthesis of complexes (I-VI) is shown in scheme 1. Yield: 62.9\%; Color: yellowish amorphous solid; $\mathbf{m p} 380{ }^{\circ} \mathrm{C}$; Mol. wt.: 578.00 g/mol; Empirical formula: $\mathrm{C}_{20} \mathrm{H}_{12} \mathrm{ClN}_{4} \mathrm{O}_{3} \mathrm{Re}$, Elemental analysis: Calc. (\%): C, 41.56; H, 2.00; N, 9.69; Re, 32.22; Found. (\%): C, 41.52; H, 1.98; N, 9.67; Re, 32.20; Conductance: $2.83 S$ $\mathrm{cm}^{2} \mathrm{~mol}^{-1}$. ${ }^{1} \mathrm{H}$ NMR (400 MHz, DMSO-d $)^{\text {) }}$ /ppm: 9.18
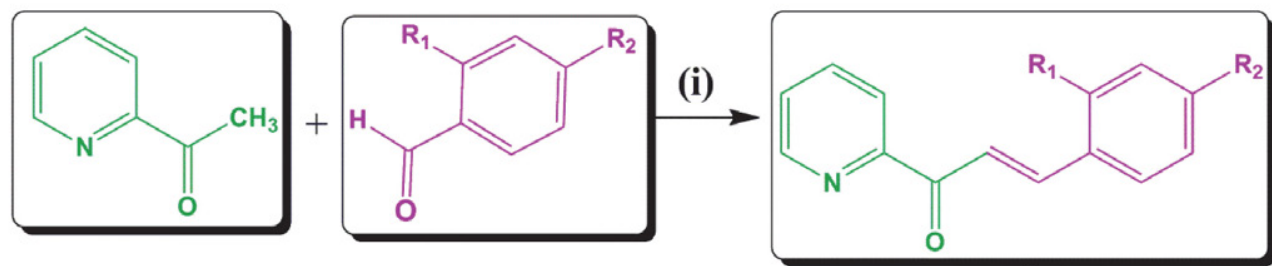

10
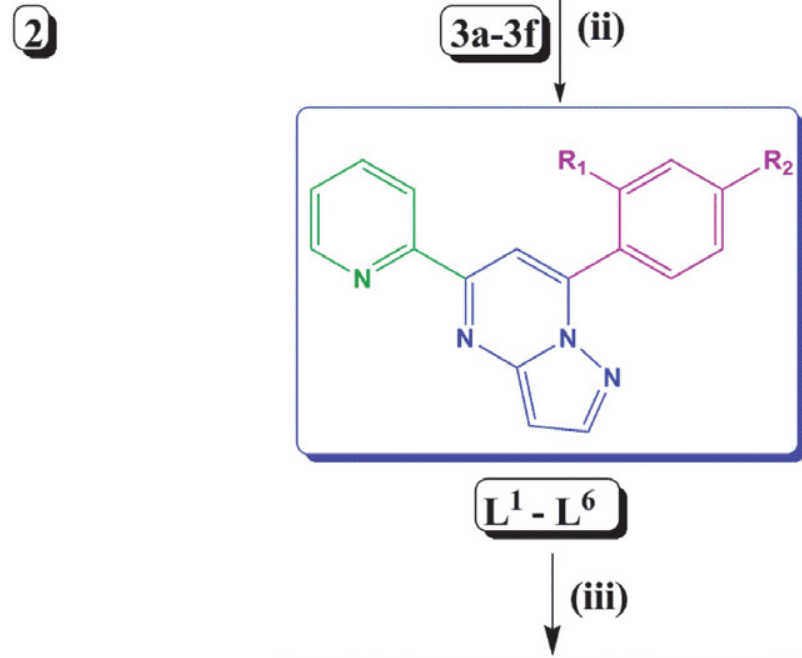

\section{Reagent and Conditions:}

(i) Methanol, $\mathrm{KOH}$

(ii) DMF, 3-amino pyrazole (4a), $\mathrm{K}^{\mathrm{t}} \mathrm{OBu}$

(iii) Ethanol, $\mathrm{Re}(\mathrm{CO}){ }_{5} \mathrm{Cl}$, reflux $60-70{ }^{\circ} \mathrm{C}$

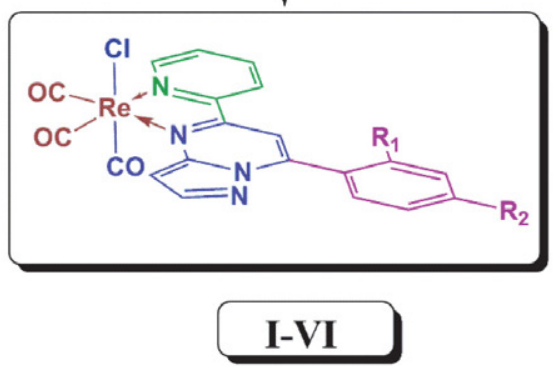

Scheme 1. Reaction scheme for the synthesis of ligands and rhenium complexes. 
$\left(2 \mathrm{H}, \mathrm{dd}, J=8.4 \mathrm{~Hz}, 6.4 \mathrm{~Hz}, \mathrm{H}_{3}\right.$ "6"), $8.60\left(1 \mathrm{H}, \mathrm{s}, \mathrm{H}_{7}\right), 8.45$ $\left(2 \mathrm{H}, \mathrm{dd}, J=11.2 \mathrm{~Hz}, 8.0 \mathrm{~Hz}, \mathrm{H}_{4}, 5\right.$, $), 8.3(2 \mathrm{H}, \mathrm{d}, J=7.6 \mathrm{~Hz}$, $\left.\mathrm{H}_{2}, 6^{\prime}\right), 7.91\left(1 \mathrm{H}, \mathrm{d}, J=6.8 \mathrm{~Hz}, \mathrm{H}_{3}\right), 7.72\left(3 \mathrm{H}, \mathrm{m}, \mathrm{H}_{3,4}, 5^{\prime}\right)$, $7.25\left(1 \mathrm{H}, \mathrm{d}, J=2.4 \mathrm{~Hz}, \mathrm{H}_{4}\right) .{ }^{13} \mathrm{C}$ NMR (100 MHz, DMSO-

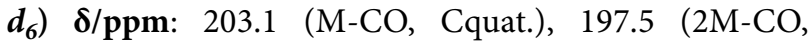
Cquat.), 157.5 ( $\mathrm{C}_{8}$, Cquat.), 154.7 ( $\mathrm{C}_{2 \text { ", }}$ Cquat.), 153.9 ( $\mathrm{C}_{6 \text { ", }}$ $-\mathrm{CH}), 149.8$ ( $\mathrm{C}_{6}$, Cquat.), 149.1 ( $\mathrm{C}_{5 \mathrm{a}}$, Cquat.), $147.1\left(\mathrm{C}_{4}\right.$ ", $-\mathrm{CH}), 140.9\left(\mathrm{C}_{3},-\mathrm{CH}\right), 132.8\left(\mathrm{C}_{3,5},-\mathrm{CH}\right), 131.0\left(\mathrm{C}_{4},-\right.$ $\mathrm{CH}), 130.0$ ( $\mathrm{C}_{1}$, Cquat.), $129.4\left(\mathrm{C}_{2}, 6,-\mathrm{CH}\right), 129.0\left(\mathrm{C}_{5}\right.$, $\mathrm{CH}), 127.6\left(\mathrm{C}_{3 "},-\mathrm{CH}\right), 106.1\left(\mathrm{C}_{7},-\mathrm{CH}\right), 99.7\left(\mathrm{C}_{4},-\mathrm{CH}\right)$. [Total signal observed $=17$ : signal of $\mathrm{C}=7(\mathrm{M}-\mathrm{CO}=2$, phenyl ring- $\mathrm{C}=1$, pyrazolo $[1,5-\mathrm{a}]$ pyrimidine- $\mathrm{C}=3$, pyridine ring- $\mathrm{C}=1$ ), signal of $\mathrm{CH}=10$ (pyrazolo[1,5-a] pyrimidine- $\mathrm{CH}=3$, phenylring- $\mathrm{CH}=3$, pyridine ring$\mathrm{CH}=4)]$; IR (KBr, 4000-400 $\left.\mathbf{~ c m}^{-1}\right)$ : 2014, $1898 v(\operatorname{Re}(-$ $\mathrm{CO}), 1550 v(\mathrm{C}=\mathrm{N}), 1504(\mathrm{C}-\mathrm{H})$ bending, $1250 v(\mathrm{C}-\mathrm{N})$, $1604 v(\mathrm{C}=\mathrm{C})$ conjugated alkenes, $763 v($ Ar-H) adjacent hydrogen.

Synthesis of $\left[\operatorname{Re}(\mathrm{CO})_{3}\left(\mathrm{~L}^{2}\right) \mathrm{Cl}\right](\mathrm{II})$ : It was synthesized using ligand $\left(\mathbf{L}^{2}\right)$ (97 $\mathrm{mg}, 0.276 \mathrm{mmol}$ ). Yield: 77.2\%; Color: yellowish amorphous solid; mp $385{ }^{\circ} \mathrm{C}$; Mol. wt.: $656.89 \mathrm{~g} / \mathrm{mol}$; Empirical formula: $\mathrm{C}_{20} \mathrm{H}_{11} \mathrm{BrClN}_{4} \mathrm{O}_{3} \mathrm{Re}$, Elemental analysis: Calc. (\%): C, 36.57; H, 1.69; N, 8.83; Re, 28.35; Found. (\%): C, 36.55; H, 1.67; N, 8.80; Re, 8.33;

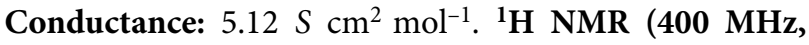
DMSO- $\left.\boldsymbol{d}_{\mathbf{6}}\right) \boldsymbol{\delta}$ /ppm: $9.16\left(2 \mathrm{H}, \mathrm{dd}, J=8.4 \mathrm{~Hz}, 7.6 \mathrm{~Hz}, \mathrm{H}_{4 ", 6}\right.$ "), $8.60\left(1 \mathrm{H}, \mathrm{s}, \mathrm{H}_{7}\right), 8.46\left(2 \mathrm{H}, \mathrm{dd}, J=6.4 \mathrm{~Hz}, 4.4 \mathrm{~Hz}, \mathrm{H}_{3,}, 5^{\prime \prime}\right)$, $8.28\left(2 \mathrm{H}, \mathrm{d}, J=8.4 \mathrm{~Hz}, \mathrm{H}_{2,6^{\prime}}\right), 7.95\left(2 \mathrm{H}, \mathrm{d}, J=8.4 \mathrm{~Hz}, \mathrm{H}_{3}\right.$, 5), $7.73\left(1 \mathrm{H}, \mathrm{d}, J=7.6 \mathrm{~Hz}, \mathrm{H}_{3}\right), 7.25\left(1 \mathrm{H}, \mathrm{d}, J=1.2 \mathrm{~Hz}, \mathrm{H}_{4}\right)$. ${ }^{13}$ C NMR (100 MHz, DMSO-d 6 ) $\delta /$ ppm: 198.9 (M-CO, Cquat.), 197.6 (2M-CO, Cquat.), 157.5 (C 6 , Cquat.), 154.6 (C2", Cquat.), 153.9 ( $\mathrm{C}_{6}$, $\left.-\mathrm{CH}\right), 149.8$ ( $\left.\mathrm{C}_{5 \mathrm{a}}, \mathrm{Cquat}.\right), 149.2$ ( $\mathrm{C}_{8}$, Cquat.), $147.1\left(\mathrm{C}_{4}\right.$, $\left.-\mathrm{CH}\right), 140.9\left(\mathrm{C}_{3},-\mathrm{CH}\right), 132.7$ $\left(\mathrm{C}_{3,5},-\mathrm{CH}\right), 132.1\left(\mathrm{C}_{2,6},-\mathrm{CH}\right), 130.9\left(\mathrm{C}_{5},-\mathrm{CH}\right), 129.5$ ( $\mathrm{C}_{1}$, Cquat.), $129.06\left(\mathrm{C}_{3}\right.$, $\left.-\mathrm{CH}\right), 126.6\left(\mathrm{C}_{4}\right.$, -Cquat. $), 106.1$ $\left(\mathrm{C}_{7},-\mathrm{CH}\right), 99.82\left(\mathrm{C}_{4},-\mathrm{CH}\right)$. [Total signal observed $=17$ : signal of $\mathrm{C}=8(\mathrm{M}-\mathrm{CO}=2$, p-Br-phenyl ring- $\mathrm{C}=2$, pyrazolo[1,5-a]pyrimidine- $\mathrm{C}=3$, pyridin ring- $\mathrm{C}=1$ ), signal of $\mathrm{CH}=9$ (pyrazolo[1,5-a]pyrimidine- $\mathrm{CH}=3, p$ - $\mathrm{Br}$ phenylring- $\mathrm{CH}=2$, pyridine ring- $\mathrm{CH}=4)$ ]; IR ( KBr, 4000-400 $\left.\mathbf{c m}^{-1}\right): 2021,1898 v(\operatorname{Re}(\mathrm{CO}), 1558 v(\mathrm{C}=\mathrm{N}), 1481(\mathrm{C}-\mathrm{H})$ bending, $1196 v(\mathrm{C}-\mathrm{N}), 1597 v(\mathrm{C}=\mathrm{C})$ conjugated alkenes, $764 v(\mathrm{Ar}-\mathrm{H})$ adjacent hydrogen.

Synthesis of $\left[\mathrm{Re}(\mathrm{CO})_{3}\left(\mathrm{~L}^{3}\right) \mathrm{Cl}\right]$ (III): It was synthesized using ligand $\left(\mathrm{L}^{3}\right)(84 \mathrm{mg}, 0.276 \mathrm{mmol})$. Yield: $140 \mathrm{mg}$, 76.1\%; Color: yellowish amorphous solid; $\mathbf{m p} 378^{\circ} \mathrm{C}$; Mol. wt.: $612.44 \mathrm{~g} / \mathrm{mol}$; Empirical formula: $\mathrm{C}_{20} \mathrm{H}_{11} \mathrm{Cl}_{2} \mathrm{~N}_{4} \mathrm{O}_{3} \mathrm{Re}$, Elemental analysis: Calc. (\%): C, 39.22; H, 1.81; N, 9.15; Re, 30.40; Found. (\%): C, 39.20; H, 1.78; N, 9.12; Re, 30.36

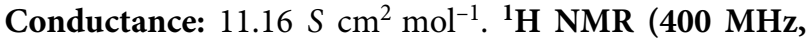

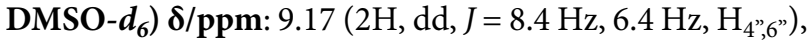
$8.61\left(1 \mathrm{H}, \mathrm{s}, \mathrm{H}_{7}\right), 8.48\left(2 \mathrm{H}, \mathrm{dd}, J=8.4 \mathrm{~Hz}, 8.0 \mathrm{~Hz}, \mathrm{H}_{3,}, 5^{\prime}\right)$, $8.36\left(2 \mathrm{H}, \mathrm{d}, J=8.8 \mathrm{~Hz}, \mathrm{H}_{2,6^{\prime}}\right), 7.91\left(1 \mathrm{H}, \mathrm{d}, J=6.4 \mathrm{~Hz}, \mathrm{H}_{3}\right)$, $7.81\left(2 \mathrm{H}, \mathrm{d}, J=8.4 \mathrm{~Hz}, \mathrm{H}_{3,5^{\prime}}\right), 7.26\left(1 \mathrm{H}, \mathrm{d}, J=2.0 \mathrm{~Hz}, \mathrm{H}_{4}\right)$.

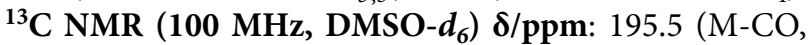
Cquat.), 189.2 (M-2CO, Cquat.), 157.5 ( $\mathrm{C}_{8}$, Cquat.), 154.7
(C2, Cquat.), 153.9 ( $\mathrm{C}_{6}$ ", $\left.-\mathrm{CH}\right), 149.3\left(\mathrm{C}_{6}\right.$, Cquat.), 148.6 ( $\mathrm{C}_{4}$, Cquat.), $147.2\left(\mathrm{C}_{4}\right.$, $\left.-\mathrm{CH}\right), 140.9\left(\mathrm{C}_{3},-\mathrm{CH}\right), 137.6$ ( $\mathrm{C}_{5 \mathrm{a}}$, Cquat.), $132.8\left(\mathrm{C}_{3,5},-\mathrm{CH}\right), 129.5\left(\mathrm{C}_{2,6},{ }^{3}, \mathrm{CH}\right), 129.2$ $\left(\mathrm{C}_{5}\right.$, $\left.-\mathrm{CH}\right), 128.7$ ( $\mathrm{C}_{1}$, Cquat.), $127.5\left(\mathrm{C}_{3}\right.$, $\left.-\mathrm{CH}\right), 106.2$ $\left(\mathrm{C}_{7},-\mathrm{CH}\right), 99.8\left(\mathrm{C}_{4},-\mathrm{CH}\right)$. [Total signal observed $=17$ : signal of $\mathrm{C}=8(\mathrm{M}-\mathrm{CO}=2, p-\mathrm{Cl}$-phenyl ring- $\mathrm{C}=2$, pyrazolo[1,5-a]pyrimidine- $\mathrm{C}=3$, pyridin ring- $\mathrm{C}=1$ ), signal of $\mathrm{CH}=9$ (pyrazolo[1,5-a]pyrimidine- $\mathrm{CH}=3, p-\mathrm{Cl}$ phenyl ring- $\mathrm{CH}=2$, pyridine ring- $\mathrm{CH}=4)$ ]; IR $(\mathbf{K B r}, \mathbf{4 0 0 0 - 4 0 0}$ $\left.\mathbf{c m}^{-1}\right):$ 2021, $1898 v(\operatorname{Re}(\mathrm{CO}), 1551 v(\mathrm{C}=\mathrm{N}), 1504(\mathrm{C}-\mathrm{H})$ bending, $1165 v(\mathrm{C}-\mathrm{N}), 1597 v(\mathrm{C}=\mathrm{C})$ conjugated alkenes, $764 v($ Ar-H $)$ adjacent hydrogen.

Synthesis of $\left[\mathrm{Re}(\mathrm{CO})_{3}\left(\mathrm{~L}^{4}\right) \mathrm{Cl}\right](\mathrm{IV})$ : It was synthesized using ligand $\left(\mathbf{L}^{\mathbf{4}}\right)(84 \mathrm{mg}, 0.276 \mathrm{mmol})$. Yield: $76.1 \%$; Color: yellowish amorphous solid; $\mathbf{m p} 368{ }^{\circ} \mathrm{C}$; Mol. wt.: $612.44 \mathrm{~g} / \mathrm{mol}$; Empirical formula: $\mathrm{C}_{20} \mathrm{H}_{11} \mathrm{Cl}_{2} \mathrm{~N}_{4} \mathrm{O}_{3} \mathrm{Re}$, Elemental analysis: Calc. (\%): C, 39.22; H, 1.81; N, 9.15; Re, 30.40, Found. (\%): C, 39.20; H, 1.78; N, 9.12; Re, 30.36;

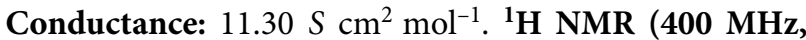

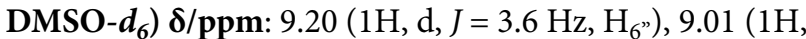
d, $J=12.8 \mathrm{~Hz}, \mathrm{H}_{4}$ ) $), 8.54\left(2 \mathrm{H}, \mathrm{d}, J=2.0 \mathrm{~Hz}, \mathrm{H}_{3}, 5^{\prime \prime}\right), 8.44$ $\left(1 \mathrm{H}, \mathrm{s}, \mathrm{H}_{7}\right), 7.89\left(2 \mathrm{H}, \mathrm{m}, \mathrm{H}_{4,5^{\prime}}\right), 7.77\left(2 \mathrm{H}, \mathrm{m}, \mathrm{H}_{3^{\prime}, 6^{\prime}}\right), 7.68$ $\left(1 \mathrm{H}, \mathrm{d}, J=7.6 \mathrm{~Hz}, \mathrm{H}_{3}\right), 7.27\left(1 \mathrm{H}, \mathrm{d}, J=2.4 \mathrm{~Hz}, \mathrm{H}_{4}\right) .{ }^{13} \mathrm{C}$

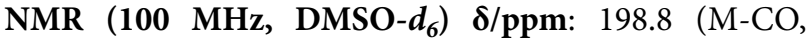
Cquat.), 197.6 (2M-CO, Cquat.), 157.6 ( $\mathrm{C}_{8}$, Cquat.), 154.5 ( $\mathrm{C}_{2 \text {, }}$, Cquat. $), 154.02\left(\mathrm{C}_{6}\right.$, $\left.-\mathrm{CH}\right), 148.40$ ( $\mathrm{C}_{6}$, Cquat. $), 147.9$ ( $\mathrm{C}_{5 \mathrm{a}}$, Cquat.), $147.3\left(\mathrm{C}_{4}\right.$, $\left.-\mathrm{CH}\right), 141.1\left(\mathrm{C}_{3},-\mathrm{CH}\right), 133.3$ $\left(\mathrm{C}_{3},-\mathrm{CH}\right), 133.1\left(\mathrm{C}_{2}\right.$, Cquat. $), 132.3\left(\mathrm{C}_{5},-\mathrm{CH}\right), 130.2\left(\mathrm{C}_{4}\right.$, $-\mathrm{CH}), 130.1$ ( $\mathrm{C}_{1}$, Cquat.), $129.6\left(\mathrm{C}_{6},-\mathrm{CH}\right), 128.1\left(\mathrm{C}_{5}\right.$, $\mathrm{CH}), 127.5\left(\mathrm{C}_{3},-\mathrm{CH}\right), 107.8\left(\mathrm{C}_{7},-\mathrm{CH}\right), 99.9\left(\mathrm{C}_{4},-\mathrm{CH}\right)$. [Total signal observed $=19$ : signal of $\mathrm{C}=8(\mathrm{M}-\mathrm{CO}=2$, $o$-Cl-phenyl ring- $\mathrm{C}=2$, pyrazolo[1,5-a]pyrimidine- $\mathrm{C}=3$, pyridin ring- $\mathrm{C}=1$ ), signal of $\mathrm{CH}=11$ (pyrazolo[1,5-a] pyrimidine- $\mathrm{CH}=3, o-\mathrm{Cl}$ phenyl ring- $\mathrm{CH}=4$, pyridine ring- $\mathrm{CH}=4)$ ]; IR (KBr, 4000-400 $\left.\mathbf{~ c m}^{-1}\right)$ : 2021, 1898 $v(\operatorname{Re}(\mathrm{CO}), 1551 v(\mathrm{C}=\mathrm{N}), 1504(\mathrm{C}-\mathrm{H})$ bending, 1165 $v(\mathrm{C}-\mathrm{N}), 1605 v(\mathrm{C}=\mathrm{C})$ conjugated alkenes, $756 v(\mathrm{Ar}-\mathrm{H}) \mathrm{ad}-$ jacent hydrogen.

Synthesis of $\left[\operatorname{Re}(\mathrm{CO})_{3}\left(L^{5}\right) \mathrm{Cl}\right](\mathrm{V})$ : It was synthesized using ligand $\left(\mathbf{L}^{5}\right)$ (84 $\mathrm{mg}, 0.276 \mathrm{mmol}$ ). Yield: 89.7\%; Color: yellowish amorphous solid; $\mathbf{m p ~} 370{ }^{\circ} \mathrm{C}$; Mol. wt.: 608.02g/mol; Empirical formula: $\mathrm{C}_{21} \mathrm{H}_{14} \mathrm{ClN}_{4} \mathrm{O}_{4} \mathrm{Re}$, Elemental analysis: Calc. (\%): C, 41.48; H, 2.32; N, 9.21; Re, 30.62, Found. (\%): C, 41.45; H, 2.30; N, 9.18; Re, 30.60; Conductance: $15.18 S \mathrm{~cm}^{2} \mathrm{~mol}^{-1}$. ${ }^{1} \mathrm{H}$ NMR (400 $\mathrm{MHz}$,

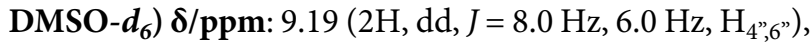
$8.60\left(1 \mathrm{H}, \mathrm{s}, \mathrm{H}_{7}\right), 8.46\left(4 \mathrm{H}, \mathrm{dd}, J=7.6 \mathrm{~Hz}, 4.8 \mathrm{~Hz}, \mathrm{H}_{2,6}, 3^{\prime}, 5^{\prime \prime}\right)$, $7.89\left(1 \mathrm{H}, \mathrm{d}, J=6.4 \mathrm{~Hz}, \mathrm{H}_{3}\right), 7.27\left(2 \mathrm{H}, \mathrm{d}, J=8.8 \mathrm{~Hz}, \mathrm{H}_{3,5}{ }^{\prime}\right)$, $7.22\left(1 \mathrm{H}, \mathrm{d}, J=2.4 \mathrm{~Hz}, \mathrm{H}_{4}\right), 3.94\left(3 \mathrm{H}, \mathrm{s},-\mathrm{OCH}_{3}\right) .{ }^{13} \mathrm{C} \mathbf{N M R}$ (100 MHz, DMSO-d $\boldsymbol{d}_{6}$ ) $/ \mathbf{p p m : ~} 199.0$ (M-CO, Cquat.), 198.2 (2M-CO, Cquat.), 163.1(C $\mathrm{C}_{4}$, Cquat.), $157.2\left(\mathrm{C}_{8}\right.$, Cquat.), 154.8 ( $\mathrm{C}_{2}$, , Cquat.), $153.8\left(\mathrm{C}_{6}\right.$, $\left.-\mathrm{CH}\right), 149.4\left(\mathrm{C}_{6}\right.$, Cquat.), $147.0\left(\mathrm{C}_{4}, \mathrm{CH}\right), 140.9\left(\mathrm{C}_{3},-\mathrm{CH}\right), 133.2\left(\mathrm{C}_{2,6},-\right.$ $\mathrm{CH}), 129.4\left(\mathrm{C}_{5},,-\mathrm{CH}\right), 127.5\left(\mathrm{C}_{3},-\mathrm{CH}\right), 123.2\left(\mathrm{C}_{5 \mathrm{a}}\right.$, -Cquat.), 121.9 ( $\mathrm{C}_{1}$, Cquat. $), 114.7\left(\mathrm{C}_{7},-\mathrm{CH}\right), 105.0\left(\mathrm{C}_{3,5}\right.$, $-\mathrm{CH}), 99.4\left(\mathrm{C}_{4},-\mathrm{CH}\right), 56.2\left(-\mathrm{OCH}_{3}\right)$. [Total signal ob- 
served $=18$ : signal of $\mathrm{C}=8\left(\mathrm{M}-\mathrm{CO}=2, p-\mathrm{OCH}_{3}\right.$-phenyl ring- $\mathrm{C}=2$, pyrazolo[1,5-a]pyrimidine- $\mathrm{C}=3$, pyridin ring- $\mathrm{C}=1$ ), signal of $\mathrm{CH}=9$ (pyrazolo[1,5-a]pyrimidine$\mathrm{CH}=3, p-\mathrm{OCH}_{3}$ phenylring $-\mathrm{CH}=2$, pyridine ring- $\mathrm{CH}=$

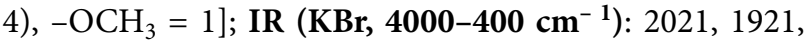
$1898 v(\operatorname{Re}(\mathrm{CO}), 1551 v(\mathrm{C}=\mathrm{N}), 1512(\mathrm{C}-\mathrm{H})$ bending, 1180 $v(\mathrm{C}-\mathrm{N}), 1597 v(\mathrm{C}=\mathrm{C})$ conjugated alkenes, $764 v(\mathrm{Ar}-\mathrm{H})$ adjacent hydrogen.

Synthesis of $\left[\operatorname{Re}(\mathrm{CO})_{3}\left(\mathrm{~L}^{6}\right) \mathrm{Cl}\right](\mathrm{VI})$ : It was synthesized using ligand $\left(\mathbf{L}^{6}\right)(79 \mathrm{mg}, 0.276 \mathrm{mmol})$. Yield: $84.9 \%$; Color: yellowish amorphous solid; $\mathbf{m p} 374{ }^{\circ} \mathrm{C}$; Mol. wt.: $592.03 \mathrm{~g} / \mathrm{mol}$; Empirical formula: $\mathrm{C}_{21} \mathrm{H}_{14} \mathrm{ClN}_{4} \mathrm{O}_{3} \mathrm{Re}$, Elemental analysis: $\mathrm{C}, 42.60 ; \mathrm{H}, 2.38 ; \mathrm{N}, 9.46 ; \mathrm{Re}, 31.45$; Found. (\%):C, 42.40; H, 2.20; N, 9.35; Re, 31.42; Conductance: $13.25 \mathrm{~S} \mathrm{~cm}^{2} \mathrm{~mol}^{-1} .{ }^{1} \mathrm{H}$ NMR $\left(400 \mathrm{MHz}\right.$, DMSO- $\left.\boldsymbol{d}_{6}\right)$

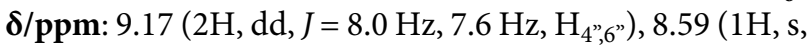
$\left.\mathrm{H}_{7}\right), 8.44\left(2 \mathrm{H}, \mathrm{dd}, J=8.4 \mathrm{~Hz}, 6.4 \mathrm{~Hz}, \mathrm{H}_{3}, 5^{\prime \prime}\right), 8.27(2 \mathrm{H}, \mathrm{d}, J$ $\left.\left.=8.0 \mathrm{~Hz}, \mathrm{H}_{2,6}\right)^{\prime}\right), 7.90\left(1 \mathrm{H}, \mathrm{d}, J=6.4 \mathrm{~Hz}, \mathrm{H}_{3}\right), 7.53(2 \mathrm{H}, \mathrm{d}, J$ $\left.=8.4 \mathrm{~Hz}, \mathrm{H}_{3,5^{\prime}}\right), 7.23\left(1 \mathrm{H}, \mathrm{d}, J=2.4 \mathrm{~Hz}, \mathrm{H}_{4}\right), 2.49(3 \mathrm{H}, \mathrm{s},-$

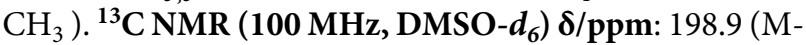
CO, Cquat.), 197.7 (2M-CO, Cquat.), 157.3 (C $\mathrm{C}_{8}$, Cquat.), 154.7 ( $\mathrm{C}_{2 \text {, }}$ Cquat.), $153.8\left(\mathrm{C}_{6}\right.$, $\left.-\mathrm{CH}\right), 149.7\left(\mathrm{C}_{6},-\right.$ Cquat.), 149.2 ( $\mathrm{C}_{5 \mathrm{a}}$, Cquat.), $147.1\left(\mathrm{C}_{4},-\mathrm{CH}\right), 143.3$ ( $\mathrm{C}_{4}$, Cquat.), $140.9\left(\mathrm{C}_{3},-\mathrm{CH}\right), 130.9\left(\mathrm{C}_{3,5},-\mathrm{CH}\right), 129.6\left(\mathrm{C}_{2,6},-\mathrm{CH}\right)$, $129.4\left(\mathrm{C}_{5},-\mathrm{CH}\right), 127.5\left(\mathrm{C}_{3},-\mathrm{CH}\right), 126.9\left(\mathrm{C}_{1}\right.$, Cquat.), $105.6\left(\mathrm{C}_{7},-\mathrm{CH}\right), 99.54\left(\mathrm{C}_{4},-\mathrm{CH}\right), 21.7\left(-\mathrm{CH}_{3}\right)$. [Total signal observed $=18$ : signal of $\mathrm{C}=8\left(\mathrm{M}-\mathrm{CO}=2, p-\mathrm{CH}_{3}-\right.$ phenyl ring- $\mathrm{C}=2$, pyrazolo $[1,5$-a]pyrimidine- $\mathrm{C}=3$, pyridine ring- $\mathrm{C}=1$ ), signal of $\mathrm{CH}=9$ (pyrazolo[1,5-a]pyrimidine- $\mathrm{CH}=3, p-\mathrm{CH}_{3}$-phenylring- $\mathrm{CH}=2$, pyridine ring- $\left.\left.\mathrm{CH}=4, \mathrm{CH}_{3}=1\right)\right]$; $\mathbf{I R}\left(\mathbf{K B r}, \mathbf{4 0 0 0}-400 \mathbf{~ c m}^{-1}\right)$ : 2021, $1913 v(\operatorname{Re}(\mathrm{CO}), 1551 v(\mathrm{C}=\mathrm{N}), 1512(\mathrm{C}-\mathrm{H})$ bending, 1196 $v(\mathrm{C}-\mathrm{N}), 1597 v(\mathrm{C}=\mathrm{C})$ conjugated alkenes, $764 v(\mathrm{Ar}-\mathrm{H})$ adjacent hydrogen.

\section{Biological activities:}

In vitro antimicrobial assay: The synthesized ligands and complexes were evaluated for their antimicrobial properties according to literature. ${ }^{17}$

In vivo brine shrimp lethality bioassay (BSLB): The brine shrimp (Artemia cysts) lethality bioassay for the synthesized compounds were carried out according to literature. ${ }^{17} 18$

Cellular level bioassay using S. cerevisiae: The in vitro cytotoxicity assay was performed in the eukaryotic system where a yeast cell, $S$. cerevisiae was taken as a model test organism. The cytotoxic effect of compounds was determined by viability staining and represented as $\%$ viability. Lower $\%$ viability indicates high toxicity of compound on that particular biological system.

Antiproliferative study: The $\operatorname{Re}(\mathrm{I})$ tricarbonyl complexes I-VI were tested for in vitro cytotoxicity against colon carcinoma (HCT116) cancerous cell lines. The MTT (3-(4,5-dimethylthiazol-2-yl)-2,5-diphenyltetrazolium bromide) assay was used to determine the cytotoxicity of the compounds. ${ }^{19}$ The extent of inhibition is displayed as an $\mathrm{IC}_{50}$ value, which is defined as the concentration required to inhibit cell growth to half. ${ }^{20,21}$ Stock solutions of $10-100 \mathrm{mg} / \mathrm{mL}$ of test complexes (I-VI) were prepared in dimethyl sulfoxide (DMSO). Twenty-four hours after cell plating, media was removed and replaced with fresh media containing $10,25,50,100,500 \mu \mathrm{g} / \mathrm{mL}$ of test compounds DMSO vehicle control, for the indicated exposure times.

DNA binding activity: Binding of metal complexes with DNA can be understood by absorption spectral analysis of DNA. The binding mode and binding constant $\left(\mathrm{K}_{\mathrm{b}}\right)$ of a complex toward DNA give an idea about the strength of interaction, which can be obtained by studying UV-Vis absorbance titration. ${ }^{22}$ The binding constant values were estimated by the following equation,

$$
\frac{[D N A]}{\left(\varepsilon_{a}-\varepsilon_{f}\right)}=\frac{[D N A]}{\left(\varepsilon_{b}-\varepsilon_{f}\right)}+\frac{1}{K_{\mathbf{b}}\left(\varepsilon_{b}-\varepsilon_{f}\right)}
$$

Where, $[\mathrm{DNA}]=$ concentration of DNA in base pairs, $\varepsilon a=$ extinction coefficient observed for the MLCT absorption band at the given DNA concentration, $\varepsilon f=$ the extinction coefficient of the complex in solution and $\varepsilon b=$ the extinction coefficient of the complex when fully bound to DNA.

Viscometric experiments were performed using Ubbelohde viscometer, maintained at $25.0( \pm 0.5){ }^{\circ} \mathrm{C}$ in a thermostatic water bath. The total system was $3 \mathrm{~mL}$, containing $100 \mu \mathrm{M}$ of DNA, and metal complexes were varied from 5 to $50 \mu \mathrm{M}$. The flow time of solutions in phosphate buffer $(\mathrm{pH}$ 7.0) was recorded, and an average flow time was calculated. Data were presented as $\left(\eta / \eta^{0}\right)^{1 / 3}$ versus [Compound]/ [DNA], where $\eta$ is the viscosity of DNA in the presence of complex and $\eta^{0}$ is the viscosity of DNA alone. All the experiment was done in triplicate. The hydrodynamic length of DNA generally increases upon partial intercalation while it does not lengthen upon groove binding. . $^{23,24}$

Molecular docking: Docking study was measured for $\operatorname{Re}(\mathrm{I})$ complexes with deoxyribonucleic acid (DNA) sequence d(ACCGACGTCGGT) $)_{2}$. The main purpose of molecular docking is to identify the binding mode of metal complexes using Hex 8.0 software. The detailed process of this study is described in literature..$^{25}$

Integrity of compounds on the DNA: For DNA integrity of compounds, the treated test organism's DNA subjected to Agarose gel electrophoresis. The DNA of S. cerevisiae was extracted according to the protocol described by Michael R. Green and Joseph Sambrook. ${ }^{26}$ The detailed process is described in literature. ${ }^{27}$

\section{Results and Discussion}

${ }^{13} \mathrm{C}$-APT, ${ }^{1} \mathrm{H}$-NMR, IR, magnetic moments, conductance measurements, and electronic spectra: The ${ }^{1} \mathrm{H}$ 
NMR spectra of ligands $\mathrm{L}^{1}-\mathrm{L}^{6}$ and complexes I-VI demonstrate peak at $6.0-8.0 \delta \mathrm{ppm}$ confirms protons of pyrazolo[1,5-a]pyrimidine aromatic ring. ${ }^{13} \mathrm{C}-\mathrm{APT}$ data of ligands $\mathrm{L}^{1}-\mathrm{L}^{6}$ and complexes I-VI show signals at 97-160 $\delta$ ppm confirm the presence of aromatic environment. ${ }^{16}$ The crystal structure of $\mathrm{Re}(\mathrm{CO})_{5} \mathrm{Cl}$ show four $\mathrm{CO}$ at equatorial position, and one $\mathrm{CO}$ along with $\mathrm{Cl}$ atom at axial position. ${ }^{28}$ The heterocyclic bidentate ligand approach from equatorial position and replace two $\mathrm{CO}$ molecules to form $\operatorname{Re}(\mathrm{I})$ complexes. In keeping with the facial arrangement of the CO ligands, the ${ }^{13} \mathrm{C}$ (APT) NMR spectra show two low-field signals in the range of 189.2-198.2 ppm and 195.5-203.1 ppm for axial and equatorial carbonyl groups of $\operatorname{Re}(\mathrm{I})$ complexes, respectively. ${ }^{29}$

Results of the FT-IR spectra of free ligands $\left(\mathrm{L}^{1}-\mathrm{L}^{6}\right)$ show the bands at $\sim 2922 \mathrm{~cm}^{-1} v(=\mathrm{C}-\mathrm{H})$ ar, and $\sim 1196 \mathrm{~cm}^{-1}$ for $-\mathrm{CN}$ stretching of pyrazolo[1,5-a]pyridine ring. The band $\sim 590-620 \mathrm{~cm}^{-1}$ is observed due to carbon-halogen bond and band at $\sim 977-1062 \mathrm{~cm}^{-1}$ is observed due to the para-substituted benzene ring. The bands at $\sim 1551$, and $\sim 1597 \mathrm{~cm}^{-1}$ are assigned to $v(\mathrm{C}=\mathrm{N})$ and $v(\mathrm{C}=\mathrm{C})$ conjugated alkene. ${ }^{27}$ In complexes, the $v(\mathrm{Re}-\mathrm{N})$ band are appeared at around $570-578 \mathrm{~cm}^{-1} .{ }^{30}$ The IR spectra of Re(I) complexes exhibit three strong $v(\mathrm{CO})$ bands in the range of 2020-1898 $\mathrm{cm}^{-1}$. ${ }^{31}$ The strong $v(\mathrm{CO})$ bands centered at $2000 \mathrm{~cm}^{-1}$ suggests expected fac-geometry around the Re metal. ${ }^{31,32}$

The observed magnetic moment values of rhenium(I) complexes are zero due to absence of unpaired electron i.e. low spin $\mathrm{t}_{2} \mathrm{~g}^{6} \mathrm{eg}^{0}$ configuration makes rhenium(I) complexes diamagnetic, and the oxidation state of rhenium is +1 in complexes.

Molar conductance values of all the low spin $\operatorname{Re}(\mathrm{I})$ complexes are found in the range of $2.83-19.25 \mathrm{~S} \mathrm{~cm}^{2} \mathrm{~mol}^{-1}$. It suggests that the $\operatorname{Re}(\mathrm{I})$ complexes are non-ionic and non-electrolytic with absence of any counter ions surrounding the coordination sphere.

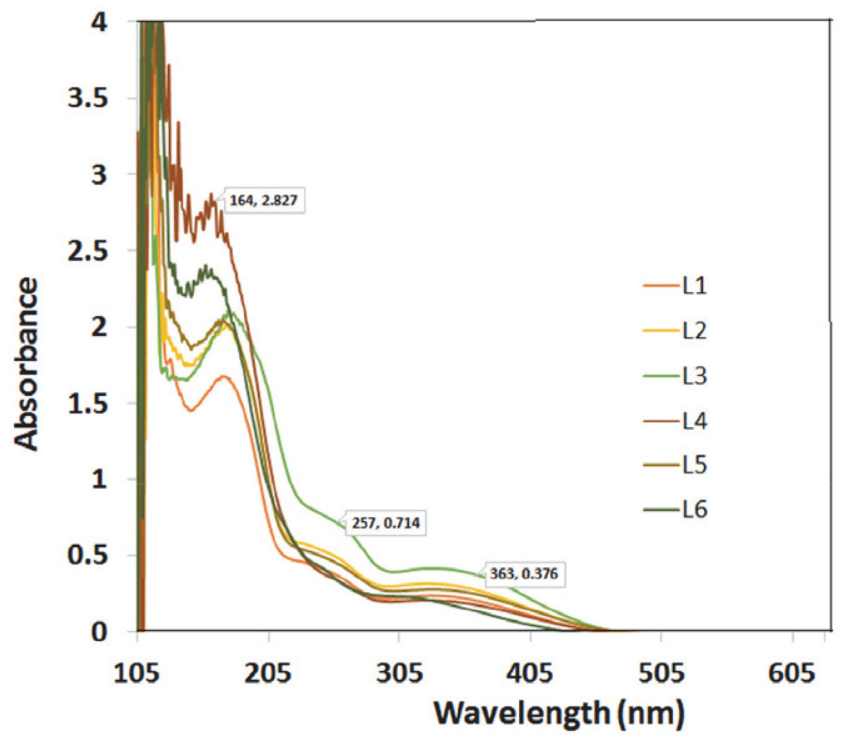

The electronic spectra of compounds were recorded in DMSO solution (Figure 1). The ground state for $t_{2} g^{6}$ electronic configuration of rhenium(I) metal ion is ${ }^{1} \mathrm{~A}_{1} \mathrm{~g}$. Three bands are observed in the electronic spectrum: one band ranging in 436.0-442.50 $\mathrm{nm}$ region assign to MLCT, second band ranging from $332.5-354.5 \mathrm{~nm}$ region attribute to $n-\pi^{*}$, and third band ranging from $286-296 \mathrm{~nm}$ assign to ultra-ligand charge transfer $\left(\pi-\pi^{\star}\right)$. It suggests that $\operatorname{Re}(\mathrm{I})$ metal complexes possess octahedral geometry. ${ }^{33}$

Biological applications of synthesized ligands and complexes:

In vitro antimicrobial screening: The data reveals that all the complexes have higher antimicrobial activity than neutral bidentate ligands and a metal salt (Figure 2). The antimicrobial activity of all complexes against different microorganisms is found better than that of the respective ligands are shown in supplementary material 3 . The MIC values of the complexes, ligands, and metal salt are observed in the range of $60-90 \mu \mathrm{M}, 280-320 \mu \mathrm{M}$, and $2500 \mu \mathrm{M}$, respectively. A comparative of antimicrobial activity (MIC) values among all synthesized metal complexes and their ligands in decreasing order are as $\mathrm{V}>\mathrm{II}$ $>\mathrm{IV}>\mathrm{VI}>\mathrm{III}>\mathrm{I}>\mathrm{L}^{5}>\mathrm{L}^{4}>\mathrm{L}^{3}>\mathrm{L}^{1}>\mathrm{L}^{2}>\mathrm{L}^{6}>\mathrm{Re}(\mathrm{CO})$ ${ }_{5} \mathrm{Cl}$ for gram positive bacteria, and $\mathrm{V}>\mathrm{IV}>\mathrm{III}>\mathrm{I}>\mathrm{VI}>$ II $>\mathrm{L}^{4}>\mathrm{L}^{5}>\mathrm{L}^{3}>\mathrm{L}^{6}>\mathrm{L}^{2}>\mathrm{L}^{1}>\mathrm{Re}(\mathrm{CO})_{5} \mathrm{Cl}$ for gram negative bacteria. The complex $\mathrm{V}$ is the most active amongst all the complexes, due to the presence of the methoxy group to the pyridine ring in pyrazolo[1,5-a]pyrimidine ligand.

The presence of a more electronegative environment in complex $\mathrm{V}$ and $\mathrm{VI}$ improves their biological properties. Two factors are applicable, that are, the ligands bound to metal ions in a multidentate fashion, and the nature of the ligand, for improving MIC values of the synthesized compounds. These may be the main reasons for the diverse antibacterial activity shown by the complexes. The pharmaco-

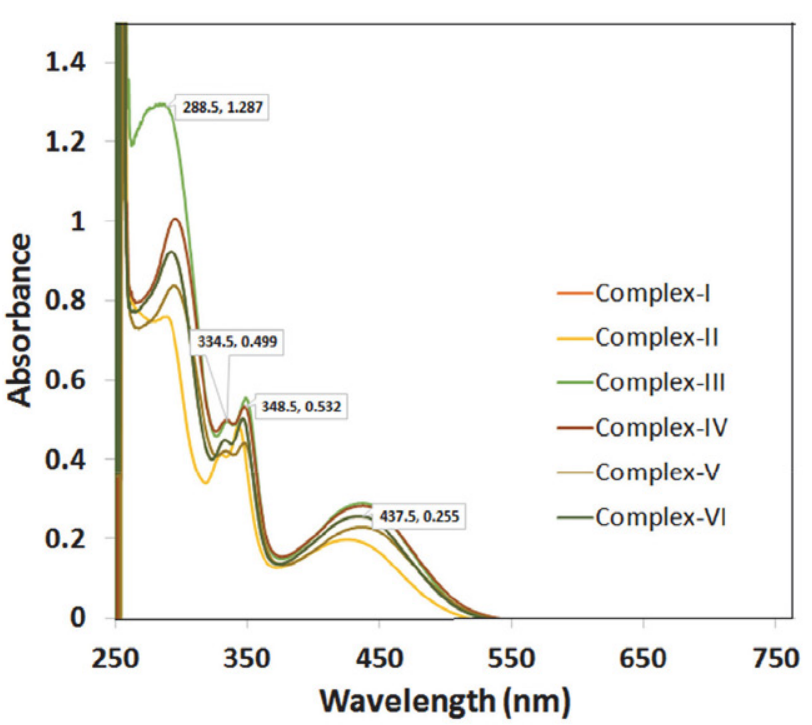

Figure 1. Electronic transition spectra of the ligands $\left(\mathrm{L}^{1}-\mathrm{L}^{6}\right)$ and complexes (I-VI). 
logical activities of these metal compounds depend on the metal ion, its ligands, and the structure of the compounds. These factors are responsible for reaching them at the proper target site in the body. It is known that certain metal ions penetrate into bacteria and inactivate their enzymes, or some metal ions can generate hydrogen peroxide, thus killing bacteria. According to overtone's concept of cell permeability, the lipid membrane that surrounds a cell favours the passage of only lipid soluble materials so that lipo-solubility is an important factor which contributes to bactericidal activity. ${ }^{34}$

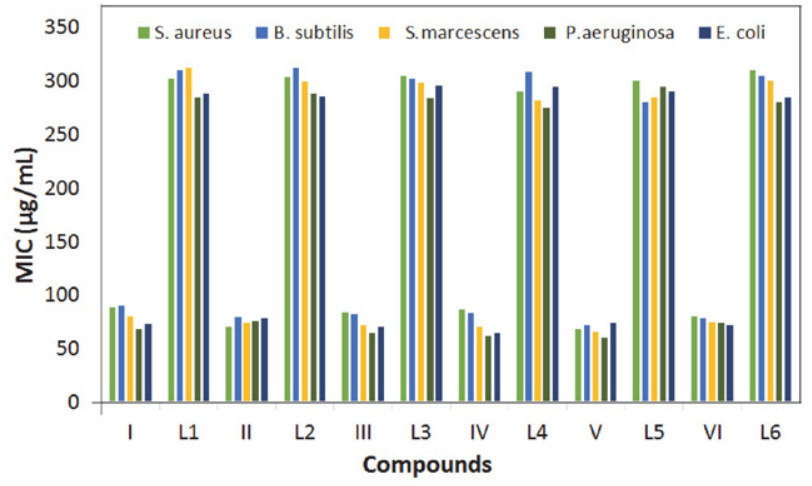

Figure 2. Antibacterial study of ligands and complexes by broth dilution method in terms of MIC in $\mu \mathrm{M}$.

Cellular level bioassay using S. cerevisiae: The in vitro cellular level cytotoxicity of ligands $\mathrm{L}^{1}-\mathrm{L}^{6}$ and complexes I-VI was found to vary with the type of substituent present in the synthesized complexes. From the results, it was found that, as the concentration of compound increases from 20 $\mu \mathrm{g} / \mathrm{mL}$ to $100 \mu \mathrm{g} / \mathrm{mL}$, cytotoxicity also increases which can be exhibited by decreasing \% viability shown in supplementary material 4 . The complexes I and II show the maximum cytotoxic effect on cells, while complexes III and IV exhibit moderate cytotoxicity, and complexes V and VI exhibit less cytotoxicity (Figure 3 and 4 ). The increasing order of $\%$ viability of ligands and complexes is $\mathrm{L}^{5}<\mathrm{L}^{3}<\mathrm{L}^{6}=\mathrm{L}^{4}<$ $\mathrm{L}^{1}<\mathrm{L}^{2}<\mathrm{V}<\mathrm{VI}<\mathrm{IV}<\mathrm{III}<\mathrm{II}<\mathrm{I}$, respectively.

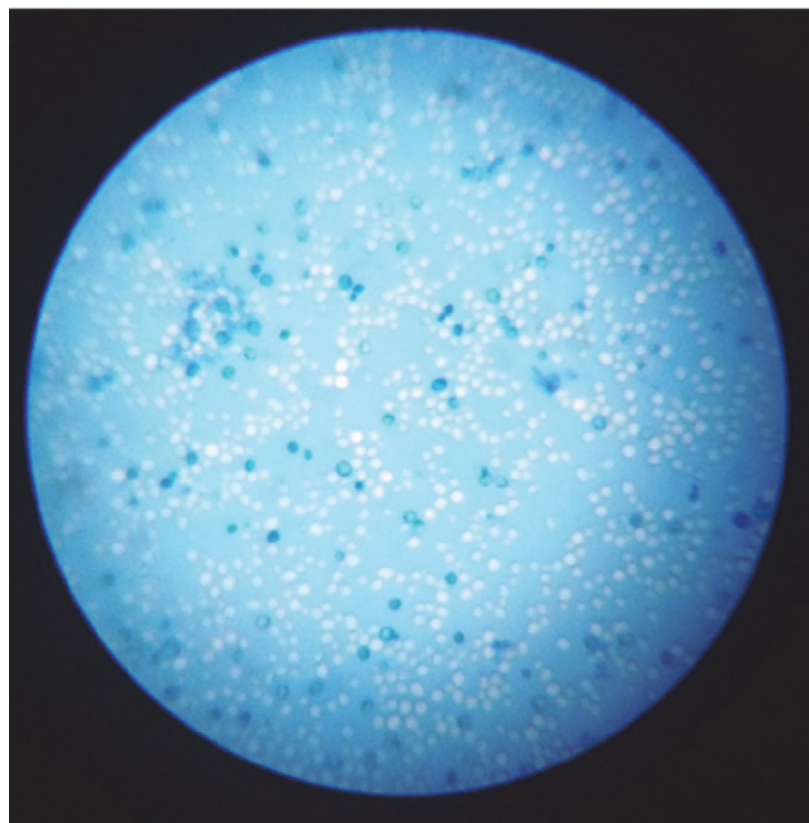

Figure 3. Cellular level cytotoxicity of synthesized compounds using S. cerevisiae, dead cells are seen dark whereas live cells are seen transparent.

In vitro brine shrimp lethality bioassay (BSLB): This method is reliable, rapid, and economical. A plot of the log of the sample's concentration versus percentage (\%) mortality of brine shrimp larvae showed a linear correlation. These results suggest that the mortality rate of brine shrimp larvae increases with increasing the concentration of the compounds. The synthesized ligands have less mortality rate as compared to the synthesized complexes. The increasing mortality rate of ligands $\left(\mathrm{LC}_{50}\right)$ and complexes $\left(\mathrm{LC}_{50}\right)$ is $\mathrm{L}^{1}(19.95)<\mathrm{L}^{3}(17.96)<\mathrm{L}^{5}(17.83)<\mathrm{L}^{4}(16.00)<$ $\mathrm{L}^{2}(11.95)<\mathrm{L}^{6}(9.84)<\mathrm{II}(9.78)<\mathrm{III}=\mathrm{V}(8.03)<\mathrm{I}(7.96)$

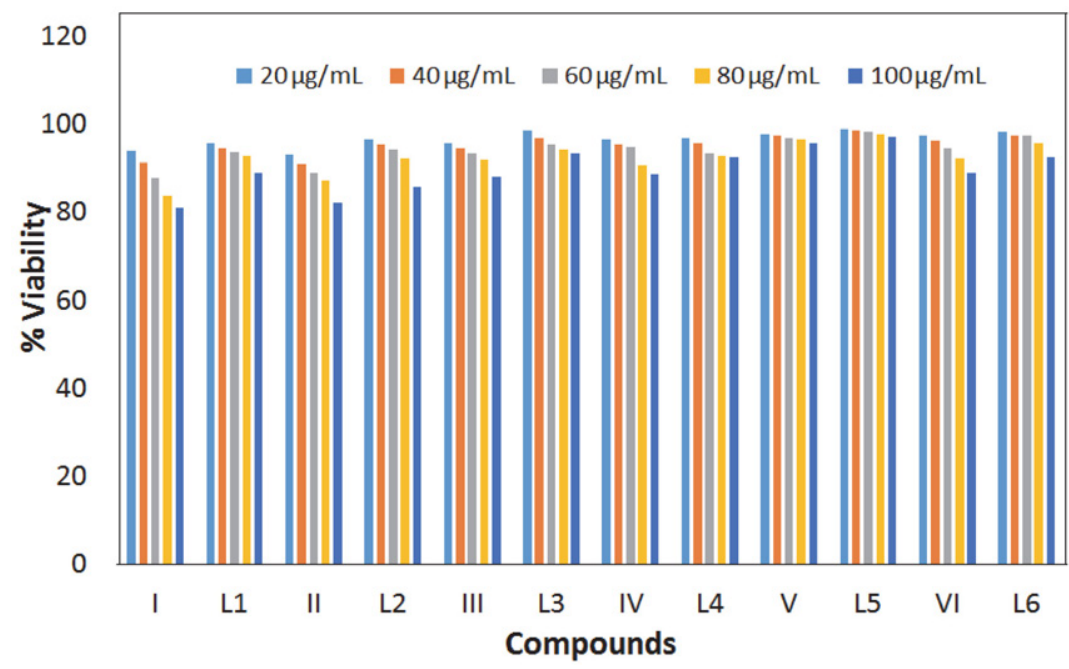

Figure 4. Effect of compounds on S. cerevisiae cells as increasing concentration. 
$<$ VI $(4.01)<$ IV (3.98). The $\mathrm{LC}_{50}$ values of the compounds are shown in brackets in $\mu \mathrm{g} / \mathrm{mL}$. Complex IV is the most potent amongst all the compounds.

DNA binding activities: Binding of metal complexes with DNA via intercalation generally results in hypochromism and a redshift (bathochromism) in the absorption band. ${ }^{35}$ Complex bind to DNA through major or minor groove results in hypochromism and redshift. The charged rhenium complex shows intercalation due to a strong stacking interaction between an aromatic moiety of the ligand and the base pair of the DNA, ${ }^{36}$ while neutral $\operatorname{Re}(\mathrm{I})$ complex shows groove binding. ${ }^{37}$ The increasing order of $\mathrm{K}_{\mathrm{b}}$ is $\mathrm{L}^{2}<\mathrm{L}^{5}<\mathrm{L}^{6}<\mathrm{II}<\mathrm{V}<\mathrm{L}^{3}<\mathrm{VI}<\mathrm{L}^{1}=\mathrm{L}^{4}<\mathrm{I}<\mathrm{IV}<$ III. The observed result shows that upon successive addition of DNA $(100 \mu L)$ at every 10 minutes time interval, a decrease in absorption intensity (hypochromism) and small redshift (1-6 nm) was observed (Figure 5). It suggests that all synthesized complexes show groove binding, which

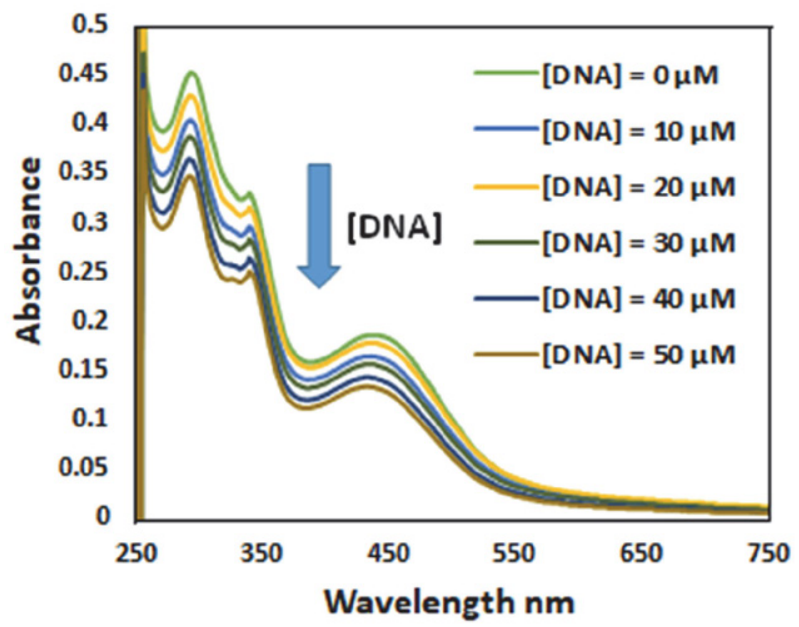

Figure 5. UV- Vis absorption spectral changes on the addition of HS DNA to the solution of complex (ligand $\mathrm{L}^{1}$ and complex I). was also confirmed by viscosity measurement and molecular docking. The organic antitumor drug netropsin has to bind within the DNA minor groove. The drug is held in place by amide hydrogen bonds to adenine N-3 and thymine $\mathrm{O}-2$ atoms. ${ }^{38}$

The binding constant $\left(\mathrm{K}_{\mathrm{b}}\right)$ values estimated from the ratio of the slop to the intercept ratio. The absorption spectral changes were monitored at around $273-296 \mathrm{~nm}$ for the investigation of the DNA binding mode and strength. As the DNA concentration was increased, the transition bands of the complexes I-VI exhibited hypochromicity [hypochromicity, $\left.\mathrm{H} \%=\left[\left(\mathrm{A}_{\text {free }}-\mathrm{A}_{\text {bound }}\right) / \mathrm{A}_{\text {free }}\right] \times 100 \%\right]$ of about $11.0-40.5 \%$, and bathochromicity of $1-6 \mathrm{~nm}$. The complex IV and the ligand $\mathrm{L}^{4}$ have the highest percentage hypochromicity (IV-28.5\%, $\mathrm{L}^{4}-40.5 \%$ ). The Gibb's free energies of the synthesized compounds are found negative values in the range of -34.30 to $-42.20 \mathrm{~kJ} \mathrm{~mol}^{-1}$ (Table 1 ). The negative value of Gibbs free energy change $\left(\Delta \mathrm{G}^{\circ}\right)$ reveals that the binding process is spontaneous.

Viscosity measurement was carried out on DNA by varying the concentration of the added $\operatorname{Re}(\mathrm{I})$ complex to get an idea of the binding mode. Groove binding typically causes less pronounced or only a minor change in the viscosity. ${ }^{39}$ The values of relative specific viscosity $\left(\boldsymbol{\eta} / \boldsymbol{\eta}^{0}\right)^{1 / 3}$ $\left\{\left(\boldsymbol{\eta}\right.\right.$ and $\left.\boldsymbol{\eta}^{0}\right)$ are the specific viscosities of DNA in the presence and absence of the $\operatorname{Re}(\mathrm{I})$ complex are plotted against $[\operatorname{Re}(\mathrm{I})$ complex] $/[\mathrm{DNA}]$ in Figure 6 . The decreasing order of the $\left(\boldsymbol{\eta} / \boldsymbol{\eta}^{0}\right)^{1 / 3}$ to the DNA is III $>$ VI $>$ II $>$ IV $>$ V $>$ I $>\mathrm{L}^{6}$ $>\mathrm{L}^{5}>\mathrm{L}^{4}>\mathrm{L}^{1}>\mathrm{L}^{2}>\mathrm{L}^{3}$, which parallels the DNA binding affinity. The increase in viscosity, observed in the presence of I-VI is small compared to the classical DNA intercalator EtBr. ${ }^{40}$ Similar enhancement in viscosity has been observed for DNA groove binding simple and mixed ligand $\mathrm{Fe}(\mathrm{II})$ and $\mathrm{Ru}$ (II) complexes containing 5,6-dmp (5,6-dimethyl-1,10-phenanthroline) as a co-ligand. ${ }^{41,42}$ The enhancement in viscosity observed in the present study is

Table 1 Binding constant $\left(\mathrm{K}_{\mathrm{b}}\right)$, percentage hypochromicity $(\% \mathrm{H})$, bathochromicity $(\Delta \lambda)$, and Gibbs free energy $\left(\Delta \boldsymbol{G}^{\circ}\right)$ values of free ligands and synthesized complexes

\begin{tabular}{|c|c|c|c|c|c|c|}
\hline \multirow[t]{2}{*}{ Compounds } & \multicolumn{2}{|c|}{$\lambda_{\max }(\mathbf{n m})$} & \multirow[t]{2}{*}{${ }^{\mathrm{a}} \Delta \lambda(\mathbf{n m})$} & \multirow{2}{*}{$\begin{array}{c}{ }^{b} K_{b} \\
\left(M^{-1}\right) \times 10^{5}\end{array}$} & \multirow[t]{2}{*}{${ }^{c} \mathrm{H} \%$} & \multirow{2}{*}{$\begin{array}{c}{ }^{\mathrm{d}} \Delta \mathrm{G}^{\mathrm{o}} \\
\left(\mathrm{Jmol}^{-1}\right)\end{array}$} \\
\hline & Free & Bound & & & & \\
\hline $\mathrm{L}^{1}$ & 277 & 278 & 1 & 1.8 & 27.8 & $-40,040.91$ \\
\hline $\mathrm{L}^{2}$ & 279 & 280 & 1 & 0.3 & 39.2 & $-34,325.59$ \\
\hline $\mathrm{L}^{3}$ & 281 & 282 & 1 & 1.3 & 30.1 & $-38,964.10$ \\
\hline $\mathrm{L}^{4}$ & 277 & 279 & 2 & 1.8 & 40.5 & $-40,040.91$ \\
\hline $\mathrm{L}^{5}$ & 276 & 277 & 1 & 0.5 & 14.9 & $-35,802.34$ \\
\hline $\mathrm{L}^{6}$ & 272 & 273 & 1 & 0.7 & 35.4 & $-36,915.72$ \\
\hline I & 292 & 294 & 2 & 2.0 & 16.8 & $-40,389.55$ \\
\hline II & 289 & 291 & 3 & 1.1 & 15.2 & $-38,411.32$ \\
\hline III & 290 & 296 & 6 & 3.5 & 16.7 & $-42,241.30$ \\
\hline IV & 291 & 295 & 4 & 3.1 & 28.5 & $-41,839.72$ \\
\hline $\mathrm{V}$ & 286 & 291 & 5 & 1.2 & 11.2 & $-38,699.24$ \\
\hline VI & 286 & 287 & 1 & 1.7 & 15.1 & $-39,851.78$ \\
\hline
\end{tabular}

${ }^{\mathrm{a}} \Delta \lambda=$ Difference between bound wavelength and free wavelength.; ${ }^{\mathrm{b}} \mathrm{K}_{\mathrm{b}}=$ Intrinsic DNA binding constant determined from the UV-visible absorption spectral titration; ${ }^{c} \mathrm{H} \%=\left[\left(\mathrm{A}_{\text {free }}-\mathrm{A}_{\text {bound }}\right) / \mathrm{A}_{\text {free }}\right] \times 100 \% ;{ }^{d} \Delta \mathrm{G}^{\circ}=$ Change in Gibb's free energy 

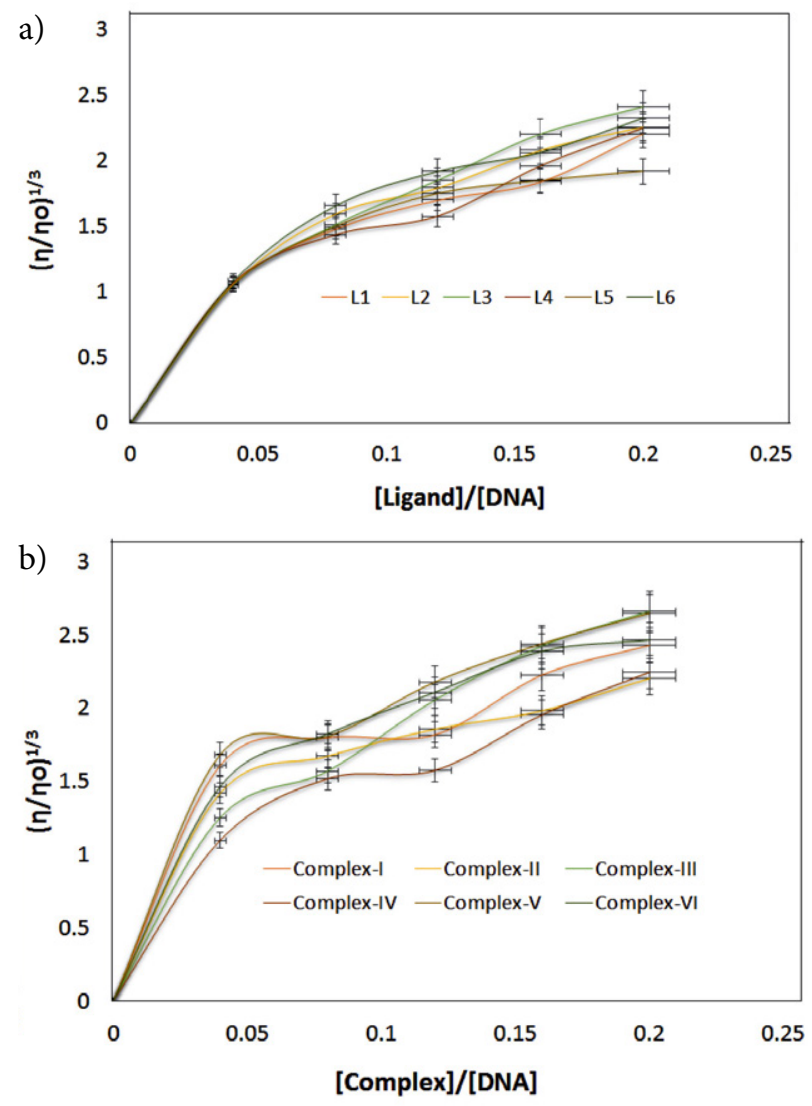

Figure 6. Effect of increasing concentration of (a) ligands and (b) complexes on the relative viscosity of HS DNA at $27( \pm 0.1){ }^{\circ} \mathrm{C}$ in phosphate buffer at $\mathrm{pH}=7.2$ :

also similar to minor groove binder netropsin. ${ }^{43}$ These show that complexes I-VI is more likely to have a DNA groove binding propensity. ${ }^{33,43}$

Molecular Docking with DNA sequence d(ACCGA CGTCGGT) $)_{2}$ : Molecular docking study is attempted to have an idea on the binding sites and favoured orientation of the ligand inside the DNA groove. ${ }^{44,45}$ The complexes and ligands are shown by the ball and stick model and DNA base pair shown by the VDW sphere using Hex 8.0 software shown in supplementary material 5 . Structure of ligands and complexes were drawn in .CDX format using ChemBioDraw Ultra 14.0 then converted to PDB format using Chem3D (Cambridge Soft). For docking studies, the structural coordinates of DNA were obtained from the protein data bank (pdb id: 423D). ${ }^{46}$ Figure 7 shows that $\operatorname{Re}(\mathrm{I})$ complexes bind with the base pair A-T, C-G, G-C, A-T (B-DNA) minor grooves of the DNA. The energy of the docked structure (I-VI and $\left.\mathrm{L}^{1}-\mathrm{L}^{6}\right)$ is $-279.72,-280.28,-283.51,-288.34,-278.84$, -281.34 , and $-233.32,-254.18,-253.77,-252.77,-251.48$, $-230.31 \mathrm{~kJ} / \mathrm{mol}$. The increasing order of energy is $\mathrm{L}^{6}<\mathrm{L}^{1}<\mathrm{L}^{5}$ $<\mathrm{L}^{4}<\mathrm{L}^{3}<\mathrm{L}^{2}<\mathrm{V}<\mathrm{I}<\mathrm{II}<\mathrm{VI}<\mathrm{III}<\mathrm{IV}$.

Effect of compounds on the integrity of DNA of S. cerevisiae cells: To determine the DNA damaging potential of the compounds a characteristic picture of comets was observed when yeast cells were exposed to increasing concentrations of compounds, increasing in smearing was observed. Agarose gel electrophoresis is a convenient method to assess the cleavage of DNA by metal-based drugs ${ }^{47}$ to determine the factors affecting the nucleolytic efficiency of a compound, and to compare the nucleolytic properties of different compounds. Figure 8 shows the electrophoretic separation of $S$. cerevisiae DNA when reacted with compounds under aerobic conditions. These clearly show that the relative binding efficacy of the complexes to DNA is much higher than the binding efficacy of pyrazolo[1,5-a]pyrimidine ligands. The difference in the DNA-cleavage efficiency of the complexes and ligands is due to the difference in binding affinity of the ligands and complexes to the DNA. In Figure 8 ligands show lesser smearing as compared to the complexes. It suggests that the cleavage efficiency of DNA is higher in the presence of complexes than the ligands. Complexes III, IV and VI show better cleavage effect of DNA, complex II shows
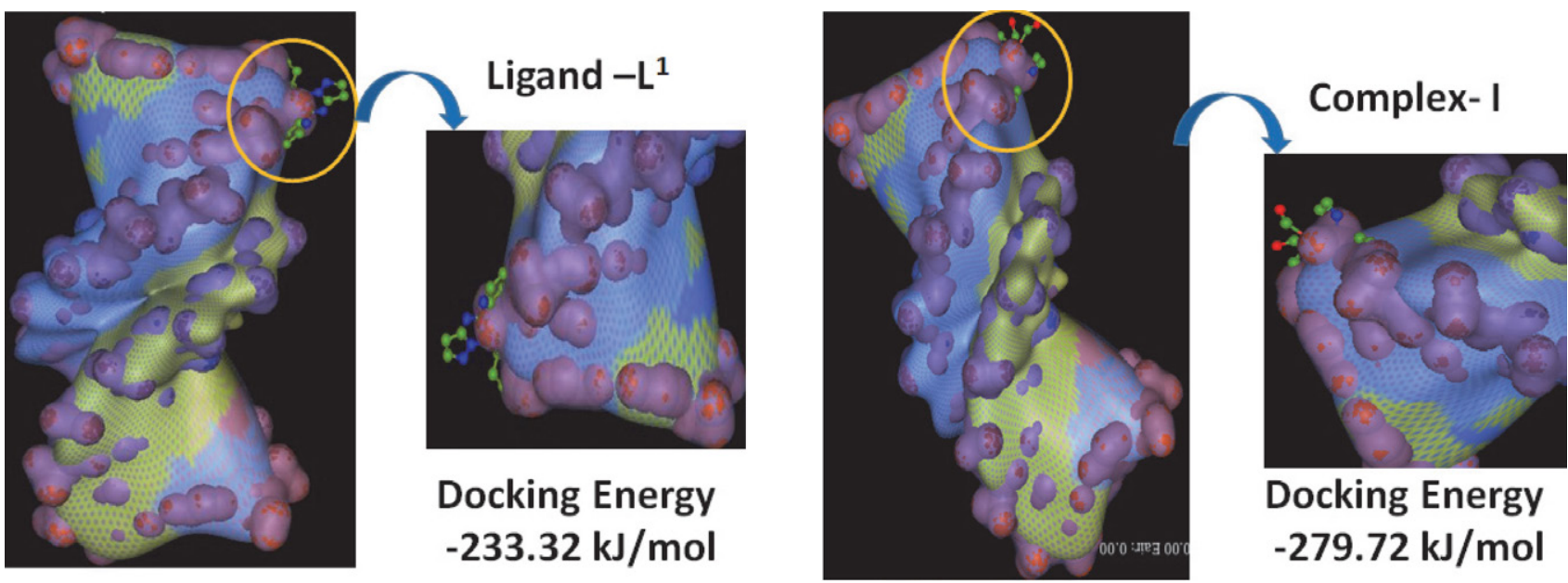

Figure 7. Molecular docking of complex I (ball and stick) with the DNA duplex (VDW spheres) of sequence d(CGCGAATTCGCG) 2 . The complex is docked inside the DNA groove. 

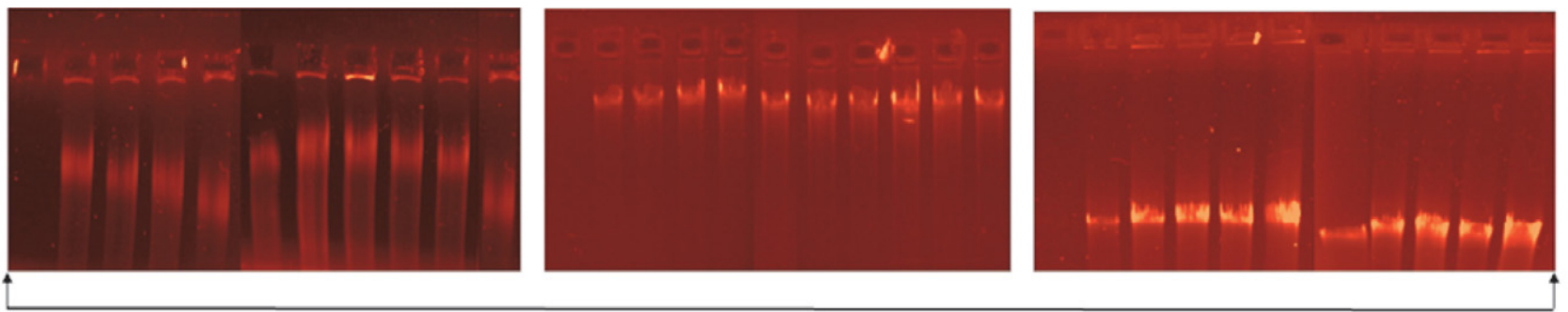

$\mathrm{L}^{1}$

I

$\mathrm{L}^{2}$

II

$L^{3}$

III
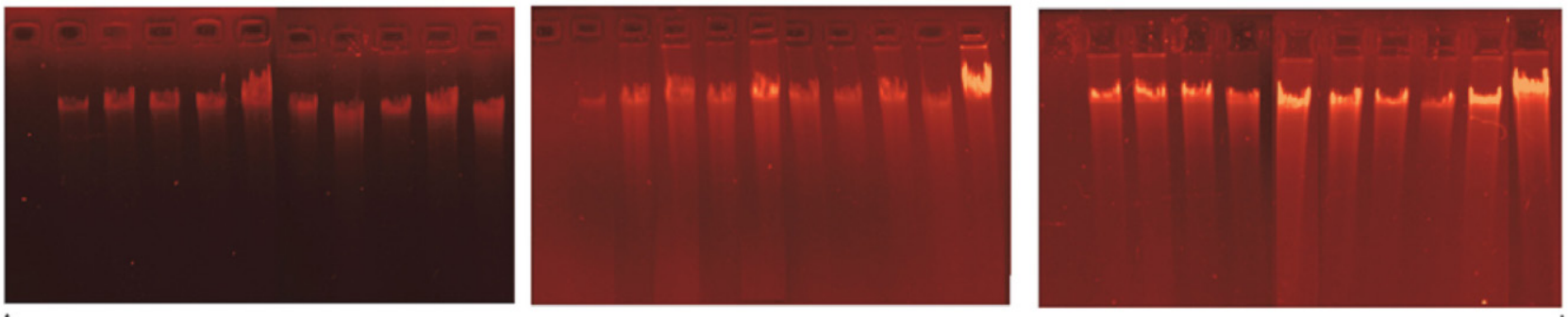

$\uparrow$

$L^{4}$

IV

$L^{5}$

V

$L^{6}$

VI

Figure 8. Photogenic view of the cleavage of S. cerevisiae DNA with a series of compounds using \% agarose gel containing $0.5 \mu \mathrm{g} / \mathrm{L}$ EtBr for $24 \mathrm{~h}$ at $37^{\circ} \mathrm{C}$.

moderate cleavage effect of DNA, and complexes I and IV show lesser cleavage effect of DNA.

Antiproliferative study: Metal carbonyls as anticancer drugs in clinical and pharmaceutical trials has wide scope because of its good solubility, and carbonyl releasing ability in the biological system. The synthesized complexes tested as MTT assay using HCT 116 cell line (Supplementary material 6). As the concentration increases the \% cell proliferation is deceases means inhibit the tumor cells. The increasing order of $\mathrm{IC}_{50}$ values is III $>$ carboplatin $>\mathrm{I}>$ oxaliplatin $>$ II $>$ cisplatin $>$ IV $=\mathrm{V}=\mathrm{VI}$. Above $500 \mu \mathrm{g} /$ $\mathrm{mL}$ concentration solution becomes turbid, coloration, and visibility not seen properly, from these, we can conclude that below $500 \mu \mathrm{g} / \mathrm{mL}$ concentration, all synthesized complexes gives good anticancer activity. The $\mathrm{IC}_{50}$ value of synthesised complex (I-VI) and standard drugs like cisplatin, carboplatin, oxaliplatin is $44.66 \mu \mathrm{g} / \mathrm{mL}, 20.50 \mu \mathrm{g} / \mathrm{mL}$, $>500 \mu \mathrm{g} / \mathrm{mL},<10 \mu \mathrm{g} / \mathrm{mL},<10 \mu \mathrm{g} / \mathrm{mL},<10 \mu \mathrm{g} / \mathrm{mL}, 15.49$ $\mu \mathrm{g} / \mathrm{mL},>111.37 \mu \mathrm{g} / \mathrm{mL}$, and $22.66 \mu \mathrm{g} / \mathrm{mL}$, respectively. The complexes IV, V, and VI are most cytotoxic than other complexes and standard drugs. The approach of metal complexes having carbon monoxide (CO) and heterocyclic compound with three to four bond distance presence of hetero atom chelated with rhenium metal is promising in terms of enhancing anticancer activity.

\section{Conclusion}

A series of substituted pyrazolo[1,5-a]pyrimidine nucleus based organometallic rhenium(I) complexes were synthesized and characterized, in search of new organometallic complexes with better antibacterial, cytotoxicity, genotoxicity, DNA binding, and DNA cleavage study. The synthesis was carried out by pentacarbonyl chloro rheni$\mathrm{um}(\mathrm{I})$ as a starting material. The spectral and analytical data are in good agreement with the proposed structure and revealed the octahedral geometry, and non-electrolytic nature of complexes. $\operatorname{Re}(\mathrm{I})$ compounds treatment to Saccharomyces cerevisiae yeast cells induced genotoxicity and changes in the conformation of cell DNA. DNA binding study was carried out by absorption titration, viscosity measurement, and molecular modelling. Binding constant $\left(\mathrm{K}_{\mathrm{b}}\right)$ values of complexes were higher than the ligands, and the studies showed groove mode of DNA binding. There was a minor change in the relative specific viscosity ( $\mathbf{\eta} /$ $\left.\boldsymbol{\eta}^{0}\right)^{1 / 3}$ ( $\boldsymbol{\eta}$ and $\boldsymbol{\eta}^{0}$ are the specific viscosities) of DNA in presence and absence of the Re(I)complex, which supports absorption spectroscopy titration data of groove mode of DNA binding. In molecular modelling, docking energies of complexes were observed higher than the ligands. The presence of a more electronegative environment improves the antibacterial activity of complexes than ligands. The increasing order of $\mathrm{LC}_{50}$ values evaluated by brine shrimp lethality bioassay is $\mathrm{L}^{1}<\mathrm{L}^{3}<\mathrm{L}^{5}<\mathrm{L}^{4}<\mathrm{L}^{2}<\mathrm{L}^{6}<\mathrm{II}<\mathrm{III}=\mathrm{V}$ $<\mathrm{I}<\mathrm{VI}<\mathrm{IV}$. All the complexes show potent in vitro cytotoxicity in cellular level bioassay compared to free ligands.

\section{Acknowledgement}

The authors are thankful to the Head, Department of Chemistry, Sardar Patel University, Vallabh Vidyanagar, 
Gujarat, India, for providing necessary research facilities, Sardar Patel University, Vallabh Vidyanagar, CPEPA, UGC, New Delhi for providing chemicals facility, DSTPURSE Sardar Patel University, Vallabh Vidyanagar for LC-MS analysis.

\section{References}

1. G. Jaouen, S. Top, A. Vessières and R. Alberto, Journal of Organometallic Chemistry 2000, 600, 23-36. DOI:10.1016/S0022-328X(00)00036-X

2. J. Wald, R. Alberto, K. Ortner and L. Candreia, Angewandte Chemie International Edition 2001, 40, 3062-3066.

DOI:10.1002/1521-3773(20010817)40:16<3062::AID-ANIE 3062>3.0.CO;2-O

3. W. H. Mahmoud, N. F. Mahmoud and G. G. Mohamed, Journal of Organometallic Chemistry 2017, 848, 288-301.

DOI:10.1016/j.jorganchem.2017.08.001

4. K. Schmidt, M. Jung, R. Keilitz, B. Schnurr and R. Gust, Inorganica Chimica Acta 2000, 306, 6-16.

DOI:10.1016/S0020-1693(00)00139-0

5. T. R. Johnson, B. E. Mann, J. E. Clark, R. Foresti, C. J. Green and R. Motterlini, Angewandte Chemie International Edition 2003, 42, 3722-3729. DOI:10.1002/anie.200301634

6. B. S. Holla, M. Mahalinga, M. S. Karthikeyan, P. M. Akberali and N. S. Shetty, Bioorganic \& medicinal chemistry 2006, 14, 2040-2047. DOI:10.1016/j.bmc.2005.10.053

7. R. Filler, Chemtech 1974, 12, 752-757.

8. M. Ghorab, Z. H. Ismail, S. M. Abdel-Gawad and A. A. Aziem, Heteroatom Chemistry 2004, 15, 57-62.

DOI:10.1002/hc. 10212

9. A. E. Rashad, M. Abdelmegid, A. H. Shamroukh and F. M. Abdelmegeid, Org. Chem. Ind. J. 2014, 10, 224-250.

10. N. Gommermann, P. Buehlmayer, A. Von Matt, W. Breitenstein, K. Masuya, B. Pirard, P. Furet, S. W. Cowan-Jacob and G. Weckbecker, Bioorganic \& medicinal chemistry letters 2010, 20, 3628-3631. DOI:10.1016/j.bmcl.2010.04.112

11. O. Fathalla, M. Zaki, S. Swelam, S. Nofal and W. El-Eraky, Acta poloniae pharmaceutica 2003, 60, 51-60.

12. S. Tzanopoulou, M. Sagnou, M. Paravatou-Petsotas, E. Gourni, G. Loudos, S. Xanthopoulos, D. Lafkas, H. Kiaris, A. Varvarigou and I. C. Pirmettis, Journal of medicinal chemistry 2010, 53, 4633-4641. DOI:10.1021/jm1001293

13. P. S. Karia, P. A. Vekariya, A. P. Patidar, D. N. Kanthecha, B. S. Bhatt and M. N. Patel, Acta Chimica Slovenica 2019, 66, 944949. DOI:10.17344/acsi.2019.5159

14. A. Guida, M. H. Lhouty, D. Tichit, F. Figueras and P. Geneste, Applied Catalysis A: General 1997, 164, 251-264.

DOI:10.1016/S0926-860X(97)00175-0

15. V. Lipson, S. Desenko, V. Borodina and M. Shirobokova, Chemistry of Heterocyclic Compounds 2007, 43, 1544-1550.

DOI:10.1007/s10593-007-0071-4

16. R. R. Varma, B. H. Pursuwani, E. Suresh, B. S. Bhatt and M. N. Patel, Journal of Molecular Structure 2020, 1200, 127068. DOI:10.1016/j.molstruc.2019.127068
17. M. N. Patel, B. S. Bhatt, P. A. Dosi, N. V. Amaravady and H. V. Movaliya, Applied Organometallic Chemistry 2012, 26, $217-$ 224. DOI:10.1002/aoc.2841

18. G. Zhao, Y. Hui, J. K. Rupprecht, J. L. McLaughlin and K. V. Wood, Journal of Natural Products 1992, 55, 347-356. DOI:10.1021/np50081a011

19. S. J. S. Franchi, R. A. de Souza, A. E. Mauro, I. Z. Carlos, L. C. de Abreu Ribeiro, F. V. Rocha and A. V. de Godoy-Netto, Acta Chimica Slovenica 2018, 65, 547-553.

DOI:10.17344/acsi.2017.4112

20. M. A. M. Basha and S. Rishikesan, Acta Chimica Slovenica 2020.

21. E. H. El-Sayed and A. A. Fadda, Acta Chimica Slovenica 2018 , 65, 853-864. DOI:10.17344/acsi.2018.4506

22. P. A. Vekariya, P. S. Karia, B. S. Bhatt and M. N. Patel, Applied Organometallic Chemistry 2019, 33, e5152.

23. F. Leng, W. Priebe and J. B. Chaires, Biochemistry 1998, 37, 1743-1753. DOI:10.1021/bi9720742

24. J. V. Mehta, S. B. Gajera, D. D. Patel and M. N. Patel, Applied Organometallic Chemistry 2015, 29, 357-367.

DOI:10.1002/aoc.3299

25. T. Sato, H. Awano, O. Haba, H. Katagiri, Y.-J. Pu, T. Takahashi and K. Yonetake, Dalton Transactions 2012, 41, 8379-8389. DOI:10.1039/c2dt30071k

26. J. Sambrook and D. W. Russell, Cold Spring Harbor Protocols 2006, 2006, pdb. prot4039. DOI:10.1101/pdb.prot3847

27. D. A. Kanthecha, B. S. Bhatt and M. N. Patel, Heliyon 2019, 5, e01968. DOI:10.1016/j.heliyon.2019.e01968

28. F. Cotton and L. Daniels, Acta Crystallographica Section C: Crystal Structure Communications 1983, 39, 1495-1496. DOI:10.1107/S0108270183009014

29. R. Kia, V. Mirkhani, A. Kálmán and A. Deák, Polyhedron 2007, 26, 1711-1716. DOI:10.1016/j.poly.2006.12.025

30 .A. Karaküçük-İyidoğan, D. Taşdemir, E. E. Oruç-Emre and J. Balzarini, European Journal of Medicinal Chemistry 2011, 46, 5616-5624. DOI:10.1016/j.ejmech.2011.09.031

31. K. Chanawanno, J. T. Engle, K. X. Le, R. S. Herrick and C. J. Ziegler, Dalton transactions 2013, 42, 13679-13684. DOI:10.1039/c3dt50894c

32. A. Núñez-Montenegro, R. Carballo and E. M. VázquezLópez, Journal of inorganic biochemistry 2014, 140, 53-63. DOI:10.1016/j.jinorgbio.2014.06.012

33. G. Balakrishnan, T. Rajendran, K. S. Murugan, M. S. Kumar, V. K. Sivasubramanian, M. Ganesan, A. Mahesh, T. Thirunalasundari and S. Rajagopal, Inorganica Chimica Acta 2015, 434, 51-59. DOI:10.1016/j.ica.2015.04.036

34. M. Rizzotto: A search for antibacterial agents, InTech, 2012.

35. F. L. Thorp-Greenwood, M. P. Coogan, L. Mishra, N. Kumari, G. Rai and S. Saripella, New Journal of Chemistry 2012, 36, 64-72. DOI:10.1039/C1NJ20662A

36. H.-K. Liu and P. J. Sadler, Accounts of Chemical Research 2011, 44, 349-359. DOI:10.1021/ar100140e

37. M. Kaplanis, G. Stamatakis, V. D. Papakonstantinou, M. Paravatou-Petsotas, C. A. Demopoulos and C. A. Mitsopoulou, Journal of inorganic biochemistry 2014, 135, 1-9.

DOI:10.1016/j.jinorgbio.2014.02.003 
38. M. L. Kopka, C. Yoon, D. Goodsell, P. Pjura and R. E. Dickerson, Journal of molecular biology 1985, 183, 553-563. DOI:10.1016/0022-2836(85)90171-8

39. S. S. Mati, S. S. Roy, S. Chall, S. Bhattacharya and S. C. Bhattacharya, The Journal of Physical Chemistry B 2013, 117, 14655-14665. DOI:10.1021/jp4090553

40. B. Nordén and F. Tjerneld, Biopolymers: Original Research on Biomolecules 1982, 21, 1713-1734.

DOI:10.1002/bip.360210904

41. P. Uma Maheswari, V. Rajendiran, H. Stoeckli-Evans and M. Palaniandavar, Inorganic chemistry 2006, 45, 37-50.

DOI:10.1021/ic050940q

42. S. Ramakrishnan, E. Suresh, A. Riyasdeen, M. A. Akbarsha and M. Palaniandavar, Dalton Transactions 2011, 40, 35243536. DOI:10.1039/c0dt00466a
43. A. K. Patra, T. Bhowmick, S. Roy, S. Ramakumar and A. R. Chakravarty, Inorganic chemistry 2009, 48, 2932-2943. DOI:10.1021/ic8017425

44. R. Bera, B. K. Sahoo, K. S. Ghosh and S. Dasgupta, International journal of biological macromolecules 2008, 42, 14-21. DOI:10.1016/j.ijbiomac.2007.08.010

45. Y. Gilad and H. Senderowitz, Journal of chemical information and modeling 2013, 54, 96-107. DOI:10.1021/ci400352t

46. D. Shao, M. Shi, Q. Zhao, J. Wen, Z. Geng and Z. Wang, Zeitschrift für anorganische und allgemeine Chemie 2015, 641, 454-459. DOI:10.1002/zaac.201400410

47. A. Pyle, J. Rehmann, R. Meshoyrer, C. Kumar, N. Turro and J. K. Barton, Journal of the American Chemical Society 1989, 111, 3051-3058. DOI:10.1021/ja00190a046

\section{Povzetek}

Sintetizirali smo nevtralne komplekse renija(I) tipa $\left[\operatorname{ReCl}(C O){ }_{3} \mathrm{~L}^{\mathrm{n}}\right]\left\{\mathrm{L}^{1}=7\right.$-fenil-5-(piridin-2-il)pirazolo[1,5-a]pirimidin, $\mathrm{L}^{2}=7$-(4-bromofenil)-5-(piridin-2-il)pirazolo[1,5-a]pirimidine, $\mathrm{L}^{3}=7$-(4-klorofenil)-5-(piridin-2-il)pirazolo[1,5-a] pirimidin, $\mathrm{L}^{4}=$ 7-(2-klorofenil)-5-(piridin-2-il)pirazolo[1,5-a]pirimidin, $\mathrm{L}^{5}=7$-(4-metoksifenil)-5-(piridin-2-il)pirazolo[1,5-a]pirimidin, $\mathrm{L}^{6}=5$-(piridin-2-il)-7-( $p$-tolil)pirazolo[1,5-a]pirimidin in jih karakterizirali s ${ }^{13} \mathrm{C}-\mathrm{APT},{ }^{1} \mathrm{H}-\mathrm{NMR}$, IR, meritvami elektronskih spektrov, magnetnimi meritvami in meritvijo predvodnosti. Anti-proliferativna aktivnost merjena na celicah HCT116 z metodo MTT nakazuje na močno citotoksično delovanje kompleksov, ki pri nekaterih presega celo aktivnost standardnih učinkovin kot so cisplatina, oksaliplatina in karboplatina. Antimikrobno delovanje kompleksov je večje kot pri pirazolo pirimidinskih ligandih. Teoretične študije interakcij med novimi spojinami in DNK smo preučevali z metodo molekularnega priklapljanja. Vrednost interakcij DNK-kompleks je med -230.31 in -288.34 $\mathrm{kJ} /$ mol. Vrednosti veznih konstant za komplekse $\left(1.1-3.5 \times 10^{5} \mathrm{M}^{-1}\right)$ so višje od vrednosti za ustrezne ligande $(0.32-1.8$ $\left.\times 10^{5} \mathrm{M}^{-1}\right)$. 